\title{
Semiparametric Transformation Models for Survival Data with a Cure Fraction
}

\author{
DONGLIN ZENG, GUOSHENG YIN, and JOSEPH G. IBRAHIM
}

We propose a class of transformation models for survival data with a cure fraction. The class of transformation models is motivated by biological considerations, and it includes both the proportional hazards and the proportional odds cure models as two special cases. An efficient recursive algorithm is proposed to calculate the maximum likelihood estimators. Furthermore, the maximum likelihood estimators for the regression coefficients are shown to be consistent and asymptotically normal, and their asymptotic variances attain the semiparametric efficiency bound. Simulation studies are conducted to examine the finite sample properties of the proposed estimators. The method is illustrated on data from a clinical trial involving the treatment of melanoma.

KEYWORDS: Cure model; Linear transformation models; Proportional hazards model; Proportional odds model; Semiparametric efficiency.

Donglin Zeng is Assistant Professor and Joseph G. Ibrahim is Professor, Department of Biostatistics, CB\# 7420, University of North Carolina, Chapel Hill, NC 27599. Guosheng Yin is Assistant Professor, Department of Biostatistics and Applied Mathematics, Unit 447, M. D. Anderson Cancer Center, The University of Texas, Houston, Texas 77030-4009. The authors thank the editor and the referees for their helpful comments and suggestions. 


\section{Introduction}

In time-to-event data arising from cancer and AIDS clinical trials, it is often observed that a proportion of subjects will never fail. For analyzing such data, cure rate models have been proposed and studied extensively. One type of commonly used cure rate model is the so-called two-component mixture cure model (Berkson and Gage, 1952), which treats the whole population as a mixture of cured subjects and non-cured subjects. This mixture model has been studied by many authors, including Gray and Tsiatis (1989), Sposto, Sather, and Baker (1992), Laska and Meisner (1992), Kuk and Chen (1992), Taylor (1995), Sy and Taylor (2000), Lu and Ying (2004) among others. The book by Maller and Zhou (1996) gives an detailed discussion of frequentist methods of inference for the two-component mixture cure model.

Although the mixture cure model is intuitively attractive, it does have several drawbacks, both from a Bayesian and frequenstist perspective, as pointed out by Chen, Ibrahim and Sinha (1999) and Ibrahim, Chen and Sinha (2001). An alternative cure rate model with desirable properties, called the promotion time cure model, has been proposed and studied by Yakovlev and Tsodikov (1996), Tsodikov (1998), and Chen, Ibrahim and Sinha (1999). In this model, the cured subjects are assumed to have survival time equal to infinity and the survival distribution for either cured subjects or noncured subjects can be integrated into one single formulation: for the $i$ th individual with covariate $\mathbf{X}_{i}$ in the population, the survival function of subject $i$ is given by

$$
S\left(t \mid \mathbf{X}_{i}\right)=\exp \left\{-\theta\left(\mathbf{X}_{i}\right) F(t)\right\}
$$

where $\theta(\cdot)$ is a known link function and $F(t)$ is a distribution function. Under the promotion time cure model (1), the cure rate is $S\left(\infty \mid \mathbf{X}_{i}\right)=\exp \left\{-\theta\left(\mathbf{X}_{i}\right)\right\}$ and the hazard rate at time $t$ for subject $i$ is equal to $\theta\left(\mathbf{X}_{i}\right) f(t)$, where $f(t)=d F(t) / d t$. Thus, we see that model (1) has the proportional hazards structure when the covariates are modeled through $\theta(\cdot)$. Moreover, when $\theta\left(\mathbf{X}_{i}\right)=\exp \left(\boldsymbol{\beta}^{T} \mathbf{X}_{i}\right)$ and $\boldsymbol{\beta}$ contains an intercept term $\beta_{0}$, model (1) becomes the usual Cox (1972) proportional hazards model subject to the restriction of a bounded cumulative baseline hazard function, given by $\Lambda(t)=$ $F(t) \exp \left(\beta_{0}\right)$. Thus, any cure rate model has a bounded cumulative hazard, leading to an improper survival function (i.e., $S(\infty)>0$ ), whereas non-cure models, such as the Cox model (Cox, 1972), have an unbounded cumulative hazard, thus leading to a proper survival function (i.e., $S(\infty)=0$ ).

Yakovlev and Tsodikov (1996) and Chen, Ibrahim and Sinha (1999) provide a biological derivation for model (1). The motivation comes from studying the time to relapse of cancer for patients with or without tumor cells. Specially, the promotion time cure model is derived as follows. For the $i$ th 
subject, let $N_{i}$ denote the number of tumor cells that have the potential of metastasizing, i.e., the number of metastasis-competent tumor cells. The $N_{i}$ 's are unobservable latent variables. We assume that $N_{i}$ has a Poisson distribution with Poisson rate (mean) $\theta\left(\mathbf{X}_{i}\right)$. We denote the promotion time for the $k$ th tumor cell by $\tilde{T}_{k}\left(k=1, \ldots, N_{i}\right)$ which is the time for the $k$ th metastasis-competent tumor cell to produce a detectable tumor mass. The $\tilde{T}_{k}$ 's are also unobservable quantities. Conditional on $N_{i}$, the $\tilde{T}_{k}$ 's are independent and identically distributed (i.i.d.) as $F$, where $F$ is sometimes referred to as the promotion time cumulative distribution function. Then the time to relapse of cancer, defined as $T=\min \left(\tilde{T}_{1}, \ldots, \tilde{T}_{N_{i}}\right)$, which is the observed event time, has the survival function

$$
\begin{aligned}
S\left(t \mid \mathbf{X}_{i}\right) & =P\left(N_{i}=0\right)+\sum_{k \geq 1} P\left(\tilde{T}_{1}>t, \ldots, \tilde{T}_{k}>t \mid N_{i}=k\right) P\left(N_{i}=k\right) \\
& =\exp \left\{-\theta\left(\mathbf{X}_{i}\right)\right\}+\sum_{k=1}^{\infty}\{1-F(t)\}^{k} \frac{\theta\left(\mathbf{X}_{i}\right)^{k} \exp \left\{-\theta\left(\mathbf{X}_{i}\right)\right\}}{k !} \\
& =\exp \left\{-\theta\left(\mathbf{X}_{i}\right) F(t)\right\} .
\end{aligned}
$$

In the derivation of (1), one critical assumption is that conditional on the number of tumor cells $N_{i}=k,\left(\tilde{T}_{1}, \ldots, \tilde{T}_{k}\right)$ are mutually independent. This assumption may be unrealistic since $\left(\tilde{T}_{1}, \ldots, \tilde{T}_{k}\right)$ are unobserved random variables taken on the same subject. One possible relaxation and remedy of this assumption is to introduce a subject-specific frailty $\xi_{i}$ such that conditional on both $N_{i}=k$ and $\xi_{i},\left(\tilde{T}_{1}, \ldots, \tilde{T}_{k}\right)$ are mutually independent with distribution function $F(t)$. Moreover, we assume that conditional on $\mathbf{X}_{i}$ and $\xi_{i}, N_{i}$ has a Poisson distribution with rate $\xi_{i} \theta\left(\mathbf{X}_{i}\right)$; thus, $\xi_{i}$ represents the heterogeneity of the Poisson rates in the $N_{i}$ 's. Following the same derivation as before, we then obtain that the survival function for the time to relapse, $T$, is

$$
S\left(t \mid \mathbf{X}_{i}\right)=E_{\xi_{i}}\left[e^{-\theta\left(\mathbf{X}_{i}\right) F(t) \xi_{i}}\right]
$$

where $E_{\xi_{i}}$ denotes the expectation with respect to $\xi_{i}$. For example, when $\xi_{i}$ has a gamma distribution with mean one, that is, $\xi_{i}$ has the density $\left\{\gamma^{1 / \gamma} \Gamma(1 / \gamma)\right\}^{-1} \xi_{i}^{1 / \gamma-1} \exp \left(-\xi_{i} / \gamma\right)$, then after simple algebra, we obtain

$$
S\left(t \mid \mathbf{X}_{i}\right)=\left\{1+\gamma \theta\left(\mathbf{X}_{i}\right) F(t)\right\}^{-1 / \gamma}
$$

Equivalently, we can write

$$
S\left(t \mid \mathbf{X}_{i}\right)=G_{\gamma}\left\{\theta\left(\mathbf{X}_{i}\right) F(t)\right\}
$$

where $G_{\gamma}(\cdot)$ is the transformation

$$
G_{\gamma}(x)= \begin{cases}(1+\gamma x)^{-1 / \gamma} & \gamma>0 \\ e^{-x} & \gamma=0\end{cases}
$$


Through (2) and (3), we obtain a very general class of transformation cure models, and note that the proportional hazards cure rate model in (1) is a special case of this class which corresponds to $\gamma=0$. There are also other interesting special cases arising from (2) and (3). When $\gamma=1$, we obtain a proportional odds type of cure model, similar in flavor to the proportional odds models with proper survival functions considered by Pettitt (1982) and Bennett (1983). Moreover, the general form of the class in (2) not only has a strong biological motivation, but also it can reduce to the usual linear transformation models studied by Cheng, Wei and Ying (1995) under a special choice of $\theta(\cdot)$. For instance, if we choose $\theta\left(\mathbf{X}_{i}\right)=\exp \left(\beta_{0}+\boldsymbol{\beta}_{1}^{T} \mathbf{Z}_{i}\right)$ with $\mathbf{X}_{i}=\left(1, \mathbf{Z}_{i}^{T}\right)^{T}, \boldsymbol{\beta}=\left(\beta_{0}, \boldsymbol{\beta}_{1}^{T}\right)^{T}$ and $\beta_{0}$ being the intercept term in the regression, then model (2) is equivalent to $S\left(t \mid \mathbf{Z}_{i}\right)=G_{\gamma}\left\{\exp \left(\boldsymbol{\beta}_{1}^{T} \mathbf{Z}_{i}\right) \Lambda(t)\right\}$ where $\Lambda(t)=F(t) \exp \left(\beta_{0}\right)$ is the cumulative baseline hazard. However, when $\theta\left(\mathbf{X}_{i}\right)$ has a form other than $\theta\left(\mathbf{X}_{i}\right)=\exp \left(\boldsymbol{\beta}^{T} \mathbf{X}_{i}\right)$, for example, if $\theta\left(\mathbf{X}_{i}\right)=\exp \left(\boldsymbol{\beta}^{T} \mathbf{X}_{i}\right) /\left\{1+\exp \left(\boldsymbol{\beta}^{T} \mathbf{X}_{i}\right)\right\}$, then model (2) is quite different from the linear transformation model.

When $\gamma$, which specifies transformations in (3), is treated as an unknown parameter, the model parameters may not be identifiable. For example, suppose that $\theta(\mathbf{X})=\exp \left(\beta_{0}\right)$. Then for any $\gamma \neq \tilde{\gamma}$, we can find a $\tilde{\beta}_{0}$, different from $\beta_{0}$, such that

$$
\left\{1+\gamma e^{\beta_{0}}\right\}^{-1 / \gamma}=\left\{1+\tilde{\gamma} e^{\tilde{\beta}_{0}}\right\}^{-1 / \tilde{\gamma}} .
$$

Thus, for any distribution function $F(t)$, we define $\tilde{F}(t)$ so that

$$
\left\{1+\gamma e^{\beta_{0}} F(t)\right\}^{-1 / \gamma}=\left\{1+\tilde{\gamma} e^{\tilde{\beta}_{0}} \tilde{F}(t)\right\}^{-1 / \tilde{\gamma}} .
$$

Clearly, $\tilde{F}(t)$ is also a distribution function. Consequently, the two sets of parameters $\left(\gamma, \beta_{0}, F\right)$ and $\left(\tilde{\gamma}, \tilde{\beta}_{0}, \tilde{F}\right)$ give the same survival function so they are not distinguishable from the observed data. More identifiability results are given in Section 4. Additionally, in most practical applications, there is little information in the data to estimate $\gamma$ with a reasonable degree of precision for small to even moderately large sample sizes. In these situations, the likelihood function of $\gamma$ is flat. Our experience shows that $\gamma$ can be well estimated when the sample size is very large, such as $n=1500$ or larger. Due to these limitations, we will focus on the $\gamma$ fixed case throughout the development of our model and asymptotic theory. However, in Section 4, we will discuss estimation of $\gamma$ when it is identifiable and also suggest a model selection strategy for choosing $\gamma$ in the $\gamma$ fixed case.

The transformation in (2) may not necessarily be from the family (3); different transformations are possible when $\xi$ takes other distributions. For example, we may consider the following Box-Cox type transformations:

$$
G_{\gamma}(x)= \begin{cases}\exp \left\{-\frac{(1+x)^{\gamma}-1}{\gamma}\right\} & \gamma>0 \\ 1 /(1+x)^{2} & \gamma=0\end{cases}
$$


In this family, $\gamma=1$ yields the proportional hazards model while $\gamma=0$ yields the proportional odds model. In this paper, we study general classes of transformations $G(\cdot)$ and link functions $\theta(\cdot)$, and examine inference based on maximum likelihood estimation. However, for ease and clarity of exposition, we will focus on the class in (3) or (4), and $\theta\left(\mathbf{X}_{i}\right)=\exp \left(\boldsymbol{\beta}^{T} \mathbf{X}_{i}\right)$ in the examples of Section 5. In addition, the promotion time cumulative distribution functions, $F(t)$, will be completely unspecified, and thus estimated nonparametrically throughout.

The rest of the paper is organized as follows. In Section 2, we introduce notation and propose an efficient computational algorithm for the maximum likelihood estimation procedure. In Section 3, we derive the asymptotic properties of the parameter estimates, including consistency and asymptotic normality. In Section 4, we discuss important issues of model selection, including estimation of $\gamma$ when it is identifiable as well as the selection of $\gamma$ when it is treated as fixed. In Section 5, we conduct simulation studies to evaluate the finite sample properties of the estimators and also illustrate the proposed model with a real dataset. Some concluding remarks are given in Section 6. Technical details for the proofs of the theorems are given in the Appendix.

\section{Maximum Likelihood Estimation}

Suppose that there are $n$ i.i.d. right-censored observations, $\left\{Y_{i}=T_{i} \wedge C_{i}, \mathbf{X}_{i}, \Delta_{i}=I\left(T_{i} \leq C_{i}\right) ; i=\right.$ $1, \ldots, n\}$, where $T_{i} \wedge C_{i}=\min \left(T_{i}, C_{i}\right)$ and $I(\cdot)$ is the indicator function. We assume that the follow-up time is infinite and a proportion of subjects never experience failure or right-censoring, that is $Y_{i}=\infty$ (so $C_{i}=\infty$ ) with probability one for some subjects. The right-censoring time $C_{i}$ is assumed to be conditionally independent of $T_{i}$ given $\mathbf{X}_{i}$ and has a finite hazard rate almost everywhere. We assume that model (2) is used to link $T_{i}$ with the covariate vector $\mathbf{X}_{i}$, where $\theta\left(\mathbf{X}_{i}\right)=\eta\left(\boldsymbol{\beta}^{T} \mathbf{X}_{i}\right), \eta(\cdot)$ is a known and strictly positive link function, and $\boldsymbol{\beta}$ includes an intercept term.

Thus, the observed-data likelihood function of the parameters $(\boldsymbol{\beta}, F)$ is given by

$$
\begin{aligned}
\prod_{i=1}^{n}\{ & {\left[\left\{-G^{\prime}\left(\eta\left(\boldsymbol{\beta}^{T} \mathbf{X}_{i}\right) F\left(Y_{i}\right)\right) \eta\left(\boldsymbol{\beta}^{T} \mathbf{X}_{i}\right) f\left(Y_{i}\right)\right\}^{\Delta_{i}}\left\{G\left(\eta\left(\boldsymbol{\beta}^{T} \mathbf{X}_{i}\right) F\left(Y_{i}\right)\right)\right\}^{\left(1-\Delta_{i}\right)}\right]^{I\left(Y_{i}<\infty\right)} } \\
& \left.\times\left[G\left(\eta\left(\boldsymbol{\beta}^{T} \mathbf{X}_{i}\right)\right)\right]^{I\left(Y_{i}=\infty\right)}\right\},
\end{aligned}
$$

where $G^{\prime}(x)$ denotes the derivative of $G$ with respect to $x$, and $f(\cdot)$ is the density function corresponding to the distribution function $F(\cdot)$ with respect to Lebesgue measure. We wish to maximize the above likelihood function to obtain the maximum likelihood estimates for $\boldsymbol{\beta}$ and $F$. However, this maximum does not exist since one can choose $f\left(Y_{i}\right)=\infty$ for some $Y_{i}$ with $\Delta_{i}=1$. Thus, we 
apply a nonparametric maximum likelihood estimation (NPMLE) approach, where $F$ is allowed to be a right-continuous function. Instead of maximizing (5), we maximize the following modified function,

$$
\begin{aligned}
\prod_{i=1}^{n}\{ & {\left[\left\{-G^{\prime}\left(\eta\left(\boldsymbol{\beta}^{T} \mathbf{X}_{i}\right) F\left(Y_{i}\right)\right) \eta\left(\boldsymbol{\beta}^{T} \mathbf{X}_{i}\right) F\left\{Y_{i}\right\}\right\}^{\Delta_{i}}\left\{G\left(\eta\left(\boldsymbol{\beta}^{T} \mathbf{X}_{i}\right) F\left(Y_{i}\right)\right)\right\}^{\left(1-\Delta_{i}\right)}\right]^{I\left(Y_{i}<\infty\right)} } \\
& \left.\times\left[G\left(\eta\left(\boldsymbol{\beta}^{T} \mathbf{X}_{i}\right)\right)\right]^{I\left(Y_{i}=\infty\right)}\right\},
\end{aligned}
$$

where $F\left\{Y_{i}\right\}$ is the jump size of $F$ at $Y_{i}$. The maximum likelihood estimate for $F$ is termed the NPMLE for $F$ and it is easy to show that the estimate for $F$ must be a distribution function only with point masses at the observed $Y_{i}$ with $\Delta_{i}=1$. In order to estimate $F(t)$ nonparametrically, we must decide upon a follow-up time such that all censored observations beyond that follow-up time, called the cure threshold, are treated as " $Y_{i}=\infty$ " (i.e., observed to be cured) and all observations lower than this threshold are treated as $Y_{i}<\infty$ (i.e., observed to be either failures or right-censored). This assumption is needed so that the model is identifiable in $(\boldsymbol{\beta}, F)$, as shown in Section 3. Note that if a parametric form is assumed for $F$, as in Ibrahim, Chen and Sinha (2001), then the condition that some of the $Y_{i}$ 's are observed to be infinity is not needed.

To compute the maximum likelihood estimates, we first derive the $F$ which maximizes (6) for fixed $\boldsymbol{\beta}$. Equivalently, we maximize the logarithm of (6) which is equal to

$$
\sum_{i=1}^{n} I\left(Y_{i}<\infty\right)\left[\Delta_{i} \log p_{i}+\Delta_{i} \log \left\{-G^{\prime}\left(\eta\left(\boldsymbol{\beta}^{T} \mathbf{X}_{i}\right) F_{i}\right)\right\}+\left(1-\Delta_{i}\right) \log G\left(\eta\left(\boldsymbol{\beta}^{T} \mathbf{X}_{i}\right) F_{i}\right)\right],
$$

subject to the constraint $\sum_{j} \Delta_{j} I\left(Y_{j}<\infty\right) p_{j}=1$, where $p_{i}=F\left\{Y_{i}\right\}$ denotes the jump size of $F$ at $Y_{i}$ and $F_{i}=\sum_{Y_{j} \leq Y_{i}, \Delta_{j}=1} p_{j}$. If we order the observed failure times from the smallest to the largest and use the indices $(1), \ldots,(m)$ for the ordered times, $Y_{(1)}<\cdots<Y_{(m)}$, where $m=\sum_{i} \Delta_{i} I\left(Y_{i}<\infty\right)$, then after introducing the Lagrange-multiplier $\lambda$, we obtain $p_{(i)}$ by solving the equation

$$
\begin{aligned}
\frac{1}{p_{(i)}}+\sum_{j=1}^{n} & \left\{\Delta_{j} \frac{G^{\prime \prime}\left(\eta\left(\boldsymbol{\beta}^{T} \mathbf{X}_{j}\right) F_{j}\right) \eta\left(\boldsymbol{\beta}^{T} \mathbf{X}_{j}\right) I\left(Y_{(i)} \leq Y_{j}<\infty\right)}{G^{\prime}\left(\eta\left(\boldsymbol{\beta}^{T} \mathbf{X}_{j}\right) F_{j}\right)}\right. \\
& \left.+\left(1-\Delta_{j}\right) \frac{G^{\prime}\left(\eta\left(\boldsymbol{\beta}^{T} \mathbf{X}_{j}\right) F_{j}\right) \eta\left(\boldsymbol{\beta}^{T} \mathbf{X}_{j}\right) I\left(Y_{(i)} \leq Y_{j}<\infty\right)}{G\left(\eta\left(\boldsymbol{\beta}^{T} \mathbf{X}_{j}\right) F_{j}\right)}\right\}-\lambda=0
\end{aligned}
$$

where $G^{\prime \prime}(x)$ denotes the second derivative of $G$ with respect to $x$. Thus, it follows that

$$
\frac{1}{p_{(i+1)}}=\frac{1}{p_{(i)}}+\sum_{Y_{(i)} \leq Y_{j}<Y_{(i+1)}}\left\{\Delta_{j} \frac{G^{\prime \prime}\left(\eta\left(\boldsymbol{\beta}^{T} \mathbf{X}_{j}\right) F_{j}\right) \eta\left(\boldsymbol{\beta}^{T} \mathbf{X}_{j}\right)}{G^{\prime}\left(\eta\left(\boldsymbol{\beta}^{T} \mathbf{X}_{j}\right) F_{j}\right)}+\left(1-\Delta_{j}\right) \frac{G^{\prime}\left(\eta\left(\boldsymbol{\beta}^{T} \mathbf{X}_{j}\right) F_{j}\right) \eta\left(\boldsymbol{\beta}^{T} \mathbf{X}_{j}\right)}{G\left(\eta\left(\boldsymbol{\beta}^{T} \mathbf{X}_{j}\right) F_{j}\right)}\right\} .
$$

Equivalently,

$$
\frac{1}{p_{(i+1)}}=\frac{1}{p_{(i)}}+\frac{G^{\prime \prime}\left(\eta\left(\boldsymbol{\beta}^{T} \mathbf{X}_{(i)}\right) F_{(i)}\right) \eta\left(\boldsymbol{\beta}^{T} \mathbf{X}_{(i)}\right)}{G^{\prime}\left(\eta\left(\boldsymbol{\beta}^{T} \mathbf{X}_{(i)}\right) F_{(i)}\right)}+\sum_{Y_{(i)}<Y_{j}<Y_{(i+1)}} \frac{G^{\prime}\left(\eta\left(\boldsymbol{\beta}^{T} \mathbf{X}_{j}\right) F_{(i)}\right) \eta\left(\boldsymbol{\beta}^{T} \mathbf{X}_{j}\right)}{G\left(\eta\left(\boldsymbol{\beta}^{T} \mathbf{X}_{j}\right) F_{(i)}\right)}
$$


where $F_{(i)}=p_{(1)}+\ldots+p_{(i)}$. Using the fact that $\sum_{i=1}^{m} p_{(i)}=1$, we can also write $(7)$ as

$$
\begin{aligned}
\frac{1}{p_{(i)}}=\frac{1}{p_{(i+1)}}-\frac{G^{\prime \prime}\left(\eta\left(\boldsymbol{\beta}^{T} \mathbf{X}_{(i)}\right)\left(1-S_{(i+1)}\right)\right) \eta\left(\boldsymbol{\beta}^{T} \mathbf{X}_{(i)}\right)}{G^{\prime}\left(\eta\left(\boldsymbol{\beta}^{T} \mathbf{X}_{(i)}\right)\left(1-S_{(i+1)}\right)\right)} & \\
& -\sum_{Y_{(i)}<Y_{j}<Y_{(i+1)}} \frac{G^{\prime}\left(\eta\left(\boldsymbol{\beta}^{T} \mathbf{X}_{j}\right)\left(1-S_{(i+1)}\right)\right) \eta\left(\boldsymbol{\beta}^{T} \mathbf{X}_{j}\right)}{G\left(\eta\left(\boldsymbol{\beta}^{T} \mathbf{X}_{j}\right)\left(1-S_{(i+1)}\right)\right)}
\end{aligned}
$$

where $S_{(i+1)}=p_{(i+1)}+p_{(i+2)}+\ldots+p_{(m)}$. From (7), we obtain a recursive formula of calculating $p_{(i+1)}$ from $p_{(i)}$ and $F_{(i)}$; while from (8), we obtain another recursive formula of calculating $p_{(i)}$ from $p_{(i+1)}$ and $S_{(i+1)}$. When $G^{\prime \prime}>0$ and $G^{\prime}<0$, we prefer to using (8) since it ensures that $0<p_{(i)}<p_{(i+1)}$ once $p_{(i+1)}>0$ and $S_{(i+1)}<1$.

Hence, from (8), we can treat $\boldsymbol{\beta}, \alpha \equiv p_{(m)}>0$ and $\lambda$ as independent parameters, and $p_{(1)}, \ldots$, $p_{(m-1)}$ are functions of $\boldsymbol{\beta}$ and $\alpha$. Then, the constrained maximum likelihood equations for $\boldsymbol{\beta}$ and $p_{(1)}, \ldots, p_{(m)}$ can be reduced to solving the following score equations for $\boldsymbol{\beta}, \alpha$ and $\lambda$,

$$
\begin{aligned}
0= & \sum_{i=1}^{m} \frac{1}{p_{(i)}} \frac{\partial}{\partial \boldsymbol{\beta}} p_{(i)}+\sum_{i=1}^{m} \frac{G^{\prime \prime}\left(\eta\left(\boldsymbol{\beta}^{T} \mathbf{X}_{(i)}\right) F_{(i)}\right)}{G^{\prime}\left(\eta\left(\boldsymbol{\beta}^{T} \mathbf{X}_{(i)}\right) F_{(i)}\right)}\left\{\eta^{\prime}\left(\boldsymbol{\beta}^{T} \mathbf{X}_{(i)}\right) \mathbf{X}_{(i)} F_{(i)}+\eta\left(\boldsymbol{\beta}^{T} \mathbf{X}_{(i)}\right) \frac{\partial}{\partial \boldsymbol{\beta}} F_{(i)}\right\} \\
& +\sum_{i=1}^{m} \sum_{Y_{(i)}<Y_{j}<Y_{(i+1)}} \frac{G^{\prime}\left(\eta\left(\boldsymbol{\beta}^{T} \mathbf{X}_{j}\right) F_{(i)}\right)}{G\left(\eta\left(\boldsymbol{\beta}^{T} \mathbf{X}_{j}\right) F_{(i)}\right)}\left\{\eta^{\prime}\left(\boldsymbol{\beta}^{T} \mathbf{X}_{j}\right) \mathbf{X}_{j} F_{(i)}+\eta\left(\boldsymbol{\beta}^{T} \mathbf{X}_{j}\right) \frac{\partial}{\partial \boldsymbol{\beta}} F_{(i)}\right\} \\
& +\sum_{j=1}^{n} \Delta_{j} \mathbf{X}_{j}+\sum_{j=1}^{n} I\left(Y_{j}=\infty\right) \frac{G^{\prime}\left(\eta\left(\boldsymbol{\beta}^{T} \mathbf{X}_{j}\right)\right)}{G\left(\eta\left(\boldsymbol{\beta}^{T} \mathbf{X}_{j}\right)\right)} \eta^{\prime}\left(\boldsymbol{\beta}^{T} \mathbf{X}_{j}\right) \mathbf{X}_{j}-\lambda \sum_{i=1}^{m} \frac{\partial}{\partial \boldsymbol{\beta}} p_{(i)}, \\
0= & \sum_{i=1}^{m} \frac{1}{p_{(i)}} \frac{\partial}{\partial \alpha} p_{(i)}+\sum_{i=1}^{m} \frac{G^{\prime \prime}\left(\eta\left(\boldsymbol{\beta}^{T} \mathbf{X}_{(i)}\right) F_{(i)}\right)}{G^{\prime}\left(\eta\left(\boldsymbol{\beta}^{T} \mathbf{X}_{(i)}\right) F_{(i)}\right)} \eta\left(\boldsymbol{\beta}^{T} \mathbf{X}_{(i)}\right) \frac{\partial}{\partial \alpha} F_{(i)} \\
& +\sum_{i=1}^{m} \sum_{Y_{(i)}<Y_{j}<Y_{(i+1)}} \frac{G^{\prime}\left(\eta\left(\boldsymbol{\beta}^{T} \mathbf{X}_{j}\right) F_{(i)}\right)}{G\left(\eta\left(\boldsymbol{\beta}^{T} \mathbf{X}_{j}\right) F_{(i)}\right)} \eta\left(\boldsymbol{\beta}^{T} \mathbf{X}_{j}\right) \frac{\partial}{\partial \alpha} F_{(i)}-\lambda \sum_{i=1}^{m} \frac{\partial}{\partial \alpha} p_{(i)}, \\
0 & \sum_{i=1}^{m} p_{(i)}-1 .
\end{aligned}
$$

After eliminating $\lambda$ from the first two equations, the Newton-Raphson algorithm can be used to solve the system of equations in (9). The first and second derivatives of $p_{(i)}$ with respect to $\boldsymbol{\beta}$ and $\alpha$ can be computed using the recursive formula (8).

We denote the maximum likelihood estimators for $\boldsymbol{\beta}$ and $\alpha$ by $\hat{\boldsymbol{\beta}}_{n}$ and $\hat{\alpha}_{n}$, respectively. We can estimate the asymptotic variance of $\left(\hat{\boldsymbol{\beta}}_{n}, \hat{\alpha}_{n}\right)$ based on the profile log-likelihood function for $(\boldsymbol{\beta}, \alpha)$, which is defined as the maximum value of the logarithm of (6) for any fixed $(\boldsymbol{\beta}, \alpha)$ and is denoted by $p l_{n}(\boldsymbol{\beta}, \alpha)$. The asymptotic variance of $\left(\hat{\boldsymbol{\beta}}_{n}, \hat{\alpha}\right)$ can be estimated using the negative inverse of the 
curvature of $p l_{n}(\boldsymbol{\beta}, \alpha)$ at $\left(\hat{\boldsymbol{\beta}}_{n}, \hat{\alpha}_{n}\right)$, i.e.,

$$
-\left.\left(\begin{array}{cc}
\frac{\partial^{2}}{\partial \boldsymbol{\beta}^{2}} p l_{n}(\boldsymbol{\beta}, \alpha) & \frac{\partial^{2}}{\partial \boldsymbol{\beta} \partial \alpha} p l_{n}(\boldsymbol{\beta}, \alpha) \\
\frac{\partial^{2}}{\partial \alpha \partial \boldsymbol{\beta}} p l_{n}(\boldsymbol{\beta}, \alpha) & \frac{\partial^{2}}{\partial \alpha^{2}} p l_{n}(\boldsymbol{\beta}, \alpha)
\end{array}\right)^{-1}\right|_{\boldsymbol{\beta}=\hat{\boldsymbol{\beta}}_{n}, \alpha=\hat{\alpha}_{n}} .
$$

Specifically, the second derivative of $p l_{n}(\boldsymbol{\beta}, \alpha)$ with respect to $\boldsymbol{\beta}$ and $\alpha$ can be calculated based on the following chain rule and the recursive formula (8):

$$
\begin{aligned}
\frac{\partial}{\partial \boldsymbol{\beta}} p l_{n}(\boldsymbol{\beta}, \alpha) & =\frac{\partial}{\partial \boldsymbol{\beta}} l_{n}(\boldsymbol{\beta}, F)+\sum_{i=1}^{m-1} \frac{\partial l_{n}(\boldsymbol{\beta}, F)}{\partial p_{(i)}} \frac{\partial p_{(i)}}{\partial \boldsymbol{\beta}}, \\
\frac{\partial}{\partial \alpha} p l_{n}(\boldsymbol{\beta}, \alpha) & =\frac{\partial}{\partial \alpha} l_{n}(\boldsymbol{\beta}, F)+\sum_{i=1}^{m-1} \frac{\partial l_{n}(\boldsymbol{\beta}, F)}{\partial p_{(i)}} \frac{\partial p_{(i)}}{\partial \alpha}
\end{aligned}
$$

where $l_{n}(\boldsymbol{\beta}, F)$ is the logarithm value of (6). The justification of the above variance estimation method is based on the profile likelihood theory in Murphy and van der Vaart (2000), and is discussed in the Appendix.

\section{Asymptotic Properties}

In this section, we establish theorems characterizing the asymptotic properties of $\left(\hat{\boldsymbol{\beta}}_{n}, \hat{\alpha}_{n}\right)$. In order to achieve consistency and asymptotic normality, we first need the following assumptions:

(C1) The covariate $\mathbf{X}$ is bounded with probability one, and if there exists a vector $\tilde{\boldsymbol{\beta}}$ such that $\tilde{\boldsymbol{\beta}}^{T} \mathbf{X}=0$ with probability one, then $\tilde{\boldsymbol{\beta}}=0$.

(C2) Conditional on $\mathbf{X}$, the right-censoring time $C$ is independent of $T$, and $P(C=\infty \mid \mathbf{X})>0$.

(C3) The true value of $\boldsymbol{\beta}$, denoted as $\boldsymbol{\beta}_{0}$, belongs to the interior of a known compact set $\mathcal{B}_{0}$, and the true promotion time cumulative distribution function $F_{0}$ is differentiable with $F_{0}^{\prime}(x)>0$ for all $x \in R^{+}$.

(C4) The link function $\eta(\cdot)$ is strictly increasing and twice-continuously differentiable with $\eta(\cdot)>0$. Furthermore, the transformation $G$ satisfies

$$
G(0)=1, G(x)>0, G^{\prime}(x)<0, G^{(3)}(x) \text { exists and is continuous, }
$$

where $G^{(3)}(x)$ is the third derivative of $G(x)$.

Condition (C1) is the usual condition for a design matrix in regression settings. The condition $P(C=\infty \mid \mathbf{X})$ in $(\mathrm{C} 2)$ ensures that at least some cured subjects are not right-censored; otherwise, if 
all subjects either fail or are right-censored, then intuitively, one would be unable to identify the cure rate. In (C3), $\boldsymbol{\beta}$ is assumed to be bounded. Such an assumption is often imposed in semiparametric inference, as practical calculation is always performed within a reasonable bounded set. Many link functions $\eta(\cdot)$ and $G(\cdot)$ satisfy the conditions in $(\mathrm{C} 4)$. Some examples of $\eta(\cdot)$ include $\eta(x)=e^{x}$, $\eta(x)=e^{x} /\left(1+e^{x}\right), \eta(x)=\Phi(x)$ where $\Phi$ is the cumulative distribution function of the standard normal distribution. Examples of transformations satisfying (C4) include the transformations $(1+\gamma x)^{-1 / \gamma}$ for $\gamma>0$ and $\exp (-x)$ for $\gamma=0$, as well as some others, such as $G(x)=\{1+\log (1+x)\}^{-\gamma}$ for $\gamma>0$ and $G(x)=\exp \left\{-\left((1+x)^{\gamma}-1\right) / \gamma\right\}$ for $\gamma>0$.

Before stating the main results, we first show that under conditions $(\mathrm{C} 1)-(\mathrm{C} 4)$, the parameters $\boldsymbol{\beta}$ and $F$ are identifiable. Suppose that two sets of parameters $(\boldsymbol{\beta}, F)$ and $(\tilde{\boldsymbol{\beta}}, \tilde{F})$ give the same likelihood function for the observed data. We claim that $\boldsymbol{\beta}=\tilde{\boldsymbol{\beta}}$ and $F=\tilde{F}$. Since

$$
\begin{gathered}
{\left[\left\{-G^{\prime}\left(\eta\left(\boldsymbol{\beta}^{T} \mathbf{X}\right) F(Y)\right) \eta\left(\boldsymbol{\beta}^{T} \mathbf{X}\right) f(Y)\right\}^{\Delta}\left\{G\left(\eta\left(\boldsymbol{\beta}^{T} \mathbf{X}\right) F(Y)\right)\right\}^{(1-\Delta)}\right]^{I(Y<\infty)}} \\
\times\left[G\left(\eta\left(\boldsymbol{\beta}^{T} \mathbf{X}\right)\right)\right]^{I(Y=\infty)} \\
=\left[\left\{-G^{\prime}\left(\eta\left(\tilde{\boldsymbol{\beta}}^{T} \mathbf{X}\right) \tilde{F}(Y)\right) \eta\left(\tilde{\boldsymbol{\beta}}^{T} \mathbf{X}\right) \tilde{f}(Y)\right\}^{\Delta}\left\{G\left(\eta\left(\tilde{\boldsymbol{\beta}}^{T} \mathbf{X}\right) \tilde{F}(Y)\right)\right\}^{(1-\Delta)}\right]^{I(Y<\infty)} \\
\times\left[G\left(\eta\left(\tilde{\boldsymbol{\beta}}^{T} \mathbf{X}\right)\right)\right]^{I(Y=\infty)},
\end{gathered}
$$

we choose $Y=\infty$. Then from the monotonicity of both $G$ and $\eta$, it follows that $\boldsymbol{\beta}^{T} \mathbf{X}=\tilde{\boldsymbol{\beta}}^{T} \mathbf{X}$. Thus, condition $(\mathrm{C} 1)$ gives $\boldsymbol{\beta}=\tilde{\boldsymbol{\beta}}$. Furthermore, by letting $\Delta=1$ and $Y=y$ and integrating both sides of (10) from 0 to $y$, we have $G\left(\eta\left(\boldsymbol{\beta}^{T} \mathbf{X}\right) F(y)\right)=G\left(\eta\left(\tilde{\boldsymbol{\beta}}^{T} \mathbf{X}\right) \tilde{F}(y)\right)$. Therefore, $F(y)=\tilde{F}(y)$.

The following theorem establishes the consistency of the maximum likelihood estimator.

Theorem 1. Under conditions $(\mathrm{C} 1)$ - (C4), with probability one,

$$
\left|\hat{\boldsymbol{\beta}}_{n}-\boldsymbol{\beta}_{0}\right| \rightarrow 0 \text { and } \sup _{t \in R^{+}}\left|\hat{F}_{n}(t)-F_{0}(t)\right| \rightarrow 0
$$

i.e., both $\hat{\boldsymbol{\beta}}_{n}$ and $\hat{F}_{n}$ are strongly consistent.

The basic idea in proving Theorem 1 is as follows: Suppose that $\hat{\boldsymbol{\beta}}_{n}$ and $\hat{F}_{n}$ converge to $\boldsymbol{\beta}^{*}$ and $F^{*}$, respectively. We first construct an empirical distribution function $\tilde{F}_{n}$ converging to $F_{0}$. Then since $\left\{l_{n}\left(\hat{\boldsymbol{\beta}}_{n}, \hat{F}_{n}\right)-l_{n}\left(\boldsymbol{\beta}_{0}, \tilde{F}_{n}\right)\right\} / n \geq 0$, where $l_{n}(\boldsymbol{\beta}, F)$ denotes the observed log-likelihood function at $(\boldsymbol{\beta}, F)$, and this difference converges to the negative Kullback-Leibler divergence between $\left(\boldsymbol{\beta}^{*}, F^{*}\right)$ and $\left(\boldsymbol{\beta}_{0}, F_{0}\right)$, the identifiability result gives $\boldsymbol{\beta}^{*}=\boldsymbol{\beta}_{0}$ and $F^{*}=F_{0}$. This establishes the consistency result in Theorem 1. Constructing the empirical function $\tilde{F}_{n}$ and using the Kullback-Leibler divergence to prove consistency has been used by many others in semiparametric theory, including Murphy (1994), Murphy, Rossini and van der Vaart (1997), Parner (1998), Slud and Vonta (2004), and Kosorok, Lee 
and Fine (2004) among others. However, observing the fact that $\hat{F}_{n}$ is a distribution function, proving the convergence of the Kullback-Leibler divergence is not trivial in our case, as shown in the Appendix.

Our second result concerns the joint asymptotic distribution of $\hat{\boldsymbol{\beta}}_{n}$ and $\hat{F}_{n}$. In order to obtain the joint asymptotic distribution for $\left(\hat{\boldsymbol{\beta}}_{n}, \hat{F}_{n}\right)$, we first introduce the set

$$
\mathcal{H}=\left\{\left(\mathbf{h}_{1}, h_{2}\right): \mathbf{h}_{1} \in R^{d},\left\|\mathbf{h}_{1}\right\|<1,\right.
$$

$h_{2}$ is a function in $[0, \infty)$ with its total variation bounded by 1$\}$.

Here, the total variation of a function $h_{2}$ is defined as the supremum of $\sum_{i=1}^{m}\left|h_{2}\left(t_{i+1}\right)-h_{2}\left(t_{i}\right)\right|$ over all finite partitions $0=t_{1}<t_{2}<\ldots<t_{m+1}=\infty$. We use $\left\|h_{2}\right\|_{V}$ to denote the total variation of $h_{2}$. Then $\sqrt{n}\left(\hat{\boldsymbol{\beta}}_{n}-\boldsymbol{\beta}_{0}, \hat{F}_{n}-F_{0}\right)$ can be treated as a linear functional in $l^{\infty}(\mathcal{H})$, the space of all bounded linear functionals on $\mathcal{H}$, which is defined as

$$
\sqrt{n}\left(\hat{\boldsymbol{\beta}}_{n}-\boldsymbol{\beta}_{0}, \hat{F}_{n}-F_{0}\right)\left[\mathbf{h}_{1}, h_{2}\right]=\sqrt{n}\left(\hat{\boldsymbol{\beta}}_{n}-\boldsymbol{\beta}_{0}\right)^{T} \mathbf{h}_{1}+\sqrt{n} \int h_{2}(t) d\left(\hat{F}_{n}-F_{0}\right) .
$$

The next theorem establishes the asymptotic distribution of $\sqrt{n}\left(\hat{\boldsymbol{\beta}}_{n}-\boldsymbol{\beta}_{0}, \hat{F}_{n}-F_{0}\right)$ in the metric space $l^{\infty}(\mathcal{H})$.

Theorem 2. Under conditions $(\mathrm{C} 1)-(\mathrm{C} 4), \sqrt{n}\left(\hat{\boldsymbol{\beta}}_{n}-\boldsymbol{\beta}_{0}, \hat{F}_{n}-F_{0}\right)$ converges weakly to a zero-mean Gaussian process in $l^{\infty}(\mathcal{H})$. Furthermore, $\hat{\boldsymbol{\beta}}_{n}$ is efficient; equivalently, its asymptotic variance attains the semiparametric efficiency bound for $\boldsymbol{\beta}_{0}$.

The covariance matrix of the asymptotic Gaussian process is given in the Appendix. The definition of the semiparametric efficiency bound can be found in Chapter 3 of Bickel, Klaassen, Ritov and Wellner (1993). Thus, Theorem 2 establishes that the maximum likelihood estimators are asymptotically normal and efficient. The proof of Theorem 2 is standard in most of the current semiparametric literature, including Murphy (1995), Parner (1998) and Kosorok et al. (2004). The proof relies on the linearization of the likelihood equations for $\hat{\boldsymbol{\beta}}_{n}$ and $\hat{F}_{n}$ and uses Theorem 3.3.1 of van der Vaart and Wellner (1996). In the proof, the verification of some Donsker classes and proving the invertibility of the information operator are the key steps. Both issues are discussed in detail in the Appendix for the proposed model.

Theorem 2 has many useful applications. By letting $h_{2}(\cdot)=I(\cdot \leq t)$ for any $t \geq 0$, we obtain that $\sqrt{n}\left(\hat{\boldsymbol{\beta}}_{n}-\boldsymbol{\beta}_{0}, \hat{F}(t)-F_{0}(t)\right)$ converges weakly to a zero-mean Gaussian process in $l^{\infty}\left(R^{d} \times[0, \infty)\right)$. As a result, for fixed $t_{0}, \sqrt{n}\left(\hat{F}_{n}\left(t_{0}\right)-F_{0}\left(t_{0}\right)\right)$ has an asymptotic normal distribution with mean zero. If its asymptotic variance can be estimated, one can easily construct a confidence interval for $F_{0}\left(t_{0}\right)$. Special choices of $t_{0}$ can be the quantiles of $F_{0}$. Furthermore, when interest is to test whether the 
true promotion distribution function is equal to a given distribution function $F_{0}$, we can construct a test statistic $\sqrt{n} \sup _{t \geq 0}\left|\hat{F}_{n}(t)-F_{0}(t)\right|$, similar to the Kolmogrov-Smirnov statistic. Then Theorem 2 implies that such a statistic has an asymptotic distribution which is the same as the supremum of a Gaussian process. We remark that in the above cases, the asymptotic covariance function of the Gaussian process in Theorem 2 needs to be estimated. One practical way of estimating this function is through a bootstrapping approach. The justification of the bootstrapping procedure can be shown using the same techniques as in Kosorok et al. (2004). We will not pursue this issue further here but rather focus only on inference for regression coefficients in the subsequent development.

\section{Estimation of the Transformation $G(\cdot)$}

In the forgoing sections, the transformation $G(\cdot)$ was assumed to be known. One important practical issue is how to estimate $G(\cdot)$ using the observed data. We discuss two possible methods to estimate this transformation.

The first approach is to consider $G(\cdot)$ from a parametric transformation family $\left\{G_{\gamma}: \gamma \in \Gamma\right\}$, where $\Gamma$ is a compact set in Euclidean space. For example, $G_{\gamma}$ arises from the family given in (3) or (4). Using the observed data, we then estimate $\gamma$ along with $\boldsymbol{\beta}$ and $F$. However, as noted in the introduction, one serious problem with this approach is the possible nonidentifiability of $\gamma$. However, for some special families of transformations, the parameters $(\gamma, \boldsymbol{\beta}, F)$ are identifiable, as given in the following proposition.

Proposition 1. Let $\mathbf{X}=\left(1, \mathbf{W}^{T}\right)^{T}$ and $\boldsymbol{\beta}_{0}$ as $\left(\beta_{01}, \boldsymbol{\beta}_{0 w}^{T}\right)^{T}$, respectively. Assume that $\mathbf{W}$ has support containing a nonempty open interior and $\boldsymbol{\beta}_{0 w}^{T} \mathbf{W} \neq 0$. Then for transformations from the family (3) and $\eta(x)=\exp (x), \boldsymbol{\beta}_{0}, F_{0}$ and $\gamma_{0}$ are identifiable.

Proof. Suppose that $(\tilde{\boldsymbol{\beta}}, \tilde{F}, \tilde{\gamma})$ gives the same observed likelihood function as $\left(\boldsymbol{\beta}_{0}, F_{0}, \gamma_{0}\right)$. That is,

$$
\begin{aligned}
& {\left[\left\{-G_{\gamma_{0}}^{\prime}\left(\eta\left(\boldsymbol{\beta}_{0}^{T} \mathbf{X}\right) F_{0}(Y)\right) \eta\left(\boldsymbol{\beta}_{0}^{T} \mathbf{X}\right) f_{0}(Y)\right\}^{\Delta}\left\{G_{\gamma_{0}}\left(\eta\left(\boldsymbol{\beta}_{0}^{T} \mathbf{X}\right) F_{0}(Y)\right)\right\}^{(1-\Delta)}\right]^{I(Y<\infty)}} \\
& \times\left[G_{\gamma_{0}}\left(\eta\left(\boldsymbol{\beta}_{0}^{T} \mathbf{X}\right)\right)\right]^{I(Y=\infty)} \\
& =\left[\left\{-G_{\tilde{\gamma}}^{\prime}\left(\eta\left(\tilde{\boldsymbol{\beta}}^{T} \mathbf{X}\right) \tilde{F}(Y)\right) \eta\left(\tilde{\boldsymbol{\beta}}^{T} \mathbf{X}\right) \tilde{f}(Y)\right\}^{\Delta}\left\{G_{\tilde{\gamma}}\left(\eta\left(\tilde{\boldsymbol{\beta}}^{T} \mathbf{X}\right) \tilde{F}(Y)\right)\right\}^{(1-\Delta)}\right]^{I(Y<\infty)} \\
& \times\left[G \tilde{\gamma}\left(\eta\left(\tilde{\boldsymbol{\beta}}^{T} \mathbf{X}\right)\right)\right]^{I(Y=\infty)},
\end{aligned}
$$

where $G_{\gamma}(x)=(1+\gamma x)^{-1 / \gamma}$. We choose $Y=\infty$ in (11) and obtain

$$
\left\{1+\tilde{\gamma} \exp \left(\tilde{\boldsymbol{\beta}}^{T} \mathbf{X}\right)\right\}^{1 / \tilde{\gamma}}=\left\{1+\gamma_{0} \exp \left(\boldsymbol{\beta}_{0}^{T} \mathbf{X}\right)\right\}^{1 / \gamma_{0}}
$$


Since both sides are analytic in $\mathbf{W}$, this equality holds for any $\mathbf{W}$ in real space. If $\gamma_{0}<\tilde{\gamma}$, then from the monotonicity of $(1+\gamma x)^{1 / \gamma}$, we have $\boldsymbol{\beta}_{0}^{T} \mathbf{X}>\tilde{\boldsymbol{\beta}}^{T} \mathbf{X}$ for any $\mathbf{X}$. Immediately, we conclude that $\tilde{\boldsymbol{\beta}}_{0 w}=\boldsymbol{\beta}_{0 w}$ and $\beta_{01}<\tilde{\beta}_{01}$. As a result, we have

$$
\left\{1+\tilde{\gamma} \exp \left(\tilde{\beta}_{01}+\boldsymbol{\beta}_{0 w}^{T} \mathbf{W}\right)\right\}^{1 / \tilde{\gamma}}=\left\{1+\gamma_{0} \exp \left(\beta_{01}+\boldsymbol{\beta}_{0 w}^{T} \mathbf{W}\right)\right\}^{1 / \gamma_{0}},
$$

and this holds for any real $\mathbf{W}$. Letting $\boldsymbol{\beta}_{0 w}^{T} \mathbf{W} \rightarrow \infty$, we then obtain $\gamma_{0}=\tilde{\gamma}$ and $\beta_{01}=\tilde{\beta}_{01}$. Furthermore, choosing $\Delta=1$ and $Y=y$ and integrating from 0 to $y$ in (11), we obtain $\tilde{F}(y)=F_{0}(y)$.

Proposition 1 states that if a continuous covariate has a non-zero effect, then $\gamma$ can be identified. When model parameters are identifiable, with some additional regularity conditions beyond (C.1)(C.4), the nonparametric maximum likelihood estimators for $\boldsymbol{\beta}, F$ and $\gamma$ are strongly consistent and asymptotically normal. The details are given in the remarks of the Appendix. This approach utilizes the observed data to estimate the transformation parameter and our proposed algorithm can be easily adapted to incorporate this extra parameter estimation. However, this approach may not be useful for practical applications for the following reasons: First, with no prior knowledge about the true covariate effects, there is always the concern regarding identifying all of the parameters in the model since nonidentifiability can cause numerical instability in the computations. Second, even if the parameters are identifiable, our experience indicates that for small samples, the likelihood function is typically quite flat as a function of $\gamma$. Thus, to obtain an accurate estimate of $\gamma$, a very large sample size is required and this may not be practical in many biomedical studies. Third, when the choices of transformations are from multiple families of transformations which are parameterized differently, this approach is no longer feasible.

Hence, we suggest the following approach for estimating the transformation $G$ in practice. When many transformations are under consideration, we can calculate the NPMLEs under each transformation then choose the transformation which maximizes the Akaike information criterion (AIC). The AIC is defined as the twice log-likelihood function minus twice the number of parameters. In some applications, to obtain algebraically simple transformations, one may also penalize the complexity of the transformation. Some possible choices of a penalty can be the maximal difference between $G(x)$ and $\exp (-x)$, so that we can choose a model close to the proportional hazards model; or, the choice can be the maximal difference between $G(x)$ and $1 /(1+x)$, so that we can choose a model close to the proportional odds model. However, the determination of the transformation complexity remains an unsolved issue so we defer further discussion to future work. Besides the AIC criterion, other criteria can also be used as well, including the Bayesian information criterion (BIC, Schwarz, 1978), the L measure (Ibrahim and Laud, 1994), or likelihood-based cross-validation. As an additional note, in 
most practice, the inference is solely based on the selected model; therefore, the variance estimate does not reflect the variation due to the model selection procedure. The correction of the variance estimate, sometimes called post-model-selection inference, is still an open problem in semiparametric inference.

In the subsequent simulation study, we will examine the performance of the NPMLEs for a fixed transformation; while, in the data application, the AIC will be used to select the best transformation to fit the data.

\section{$5 \quad$ Numerical Studies}

\subsection{Simulation}

We conducted simulation studies to examine the small-sample performance of our proposed methodology. In the first simulation study, the transformation cure model had survival function of the form

$$
S\left(t \mid X_{1}, X_{2}\right)=\left\{1+\gamma \exp \left(\beta_{0}+\beta_{1} X_{1}+\beta_{2} X_{2}\right) F(t)\right\}^{-1 / \gamma},
$$

where $X_{1}$ was a uniformly distributed random variable in $[0,1], X_{2}$ was a Bernoulli random variable, $\beta_{0}=0.5, \beta_{1}=1, \beta_{2}=-0.5$, and $F(t)=1-\exp (-t)$. We chose $\gamma$ to vary from 0 to 1 . Moreover, each subject had a $40 \%$ chance of being right-censored and the censoring time was generated from an exponential distribution with mean 1. The censoring proportions varied from $17 \%$ to $22 \%$ as $\gamma$ changed from 0 to 1 ; while the cure rate could be as low as $8 \%$ when $\gamma=0$ and became $20 \%$ when $\gamma=1$. For each simulated dataset, the proposed method of Section 2 was implemented to calculate the maximum likelihood estimates of $\boldsymbol{\beta}$ and its corresponding variance estimate. In solving the score equations using the Newton-Raphson iterations, the initial values for $\boldsymbol{\beta}$ were set to zero and the initial value for $\alpha$ was set to $1 / n$, with $n$ being the sample size. Other initial values were also tested in the simulation study and results were very robust to those choices. The convergence of each simulation was fast and often obtained within ten iterations.

Table 1 summarizes the results from 1000 replications for each combination of $\gamma$ and $n$ : the column labeled "Estimate" denotes the average values of the estimates; "SE" is the sample standard error of the estimates; "ESE" is the average of the estimated standard errors; and "CP" is the coverage proportion of $95 \%$ confidence intervals constructed based on the asymptotic normal approximation. The results in Table 1 indicate that the proposed estimation method performs well with sample sizes of 100 and 200: the biases are small, the estimated standard errors agree well with the sample standard errors, and the coverage probabilities are accurate. 
In the second simulation study, we generated the failure time from the transformation cure model with survival function

$$
S\left(t \mid X_{1}, X_{2}\right)=\exp \left[-\left\{\left(1+\gamma \exp \left(\beta_{0}+\beta_{1} X_{1}+\beta_{2} X_{2}\right) F(t)\right)^{\gamma}-1\right\} / \gamma\right]
$$

where $F(t)=1-\exp (-t)$ and the covariates and censoring time were generated using the same distributions as in the first simulation. In this setting, we also varied $\gamma$ from 0 to 1 , where $\gamma=0$ corresponds to the proportional odds cure model and $\gamma=1$ corresponds to the proportional hazards cure model. The censoring proportion and the cure rate were $22 \%$ and $20 \%$ when $\gamma=0$ and became $17 \%$ and $8 \%$ when $\gamma=1$. The results based on 1000 repetitions for sample sizes 100 and 200 are summarized in Table 2. From Table 2, we obtain the same conclusions as in the first simulation study. Thus, we conclude that the maximum likelihood estimation procedure proposed here not only provides an asymptotically efficient estimator, but also yields good inferential properties for small sample sizes.

Since the proportional hazards cure model and the proportional odds cure model are commonly used in practice, we also conducted a simulation study to examine the performance of the estimates based on these two models when data were generated from a different model. Specifically, we utilized the same setting for generating the covariates and censoring time as in the other two simulations described above, while we generated the survival time either from the model with a transformation $(1+x / 2)^{-2}$ or $\exp \left\{-2\left((1+x)^{1 / 2}-1\right)\right\}$; equivalently, $\gamma=1 / 2$ in both classes of (3) and (4). Both choices corresponded to a model between the proportional hazards cure model and the proportional odds cure model. The results based on 1000 replications are reported in Table 3. From Table 3, we observe that both the proportional hazards cure model and proportional odds cure models produce notable bias. Interestingly, both models estimate the direction of the coefficients correctly and the proportional hazards cure model tends to bias towards zero while the opposite is observed for the proportional odds cure model. The bias for the intercept term in both models is large but the bias for other covariate effects are relatively small. We also observe that even with sizable bias, standard error estimates of the regression coefficients corresponding to the covariates appear to be correct.

Finally, we considered the estimation of $\gamma$. We generated failure times using the cure model for the transformation class $G(x)=(1+\gamma x)^{-1 / \gamma}$. The simulation study, which is not shown here, indicates that the performance of the NPMLEs is poor and the convergence in calculating the NPMLEs is often problematic with a sample size of $n=400$. This is due to the fact that the likelihood function tends to be flat when $\gamma$ varies around the true value. 


\subsection{Application to Melanoma Data}

As an illustration, we applied the transformation cure model in (2) to a phase III melanoma clinical trial conducted by the Eastern Cooperative Oncology Group (ECOG), labeled E1690 (Kirkwood et al., 2000). This trial consisted of two treatment arms with a total of $n=427$ patients on the combined treatment arms, of which 241 patients experienced the event (cancer relapse). The response variable was relapse-free survival (RFS) time (in years). The covariates included in this analysis were treatment (high-dose interferon=1, observation=0), age (a continuous variable which ranged from 19.13 to 78.05 with a mean of 47.93 years), sex $($ female $=1$, male $=0$ ) and nodal category (taking a value of 0 if there were zero positive nodes, or 1 if there were one or more positive nodes). The median follow-up time for this study was 4.33 years, which is considered as a sufficient length of follow-up for this disease. The solid and dotted curves in Figure 1 show the Kaplan-Meier survival curves for the two treatment arms. We see that a reasonable plateau has been reached at the tails of the survival curves, and it appears that based on this period of follow-up, a cure rate model would be a suitable approach for the data. Cure rate models for the E1690 data were also considered in Chen, Harrington and Ibrahim (2002), and were shown to fit better than proper survival models. Based on Figure 1, we considered the subjects as "cured" if they were censored at 5.5 years or beyond. In the dataset, 30 subjects had censored RFS times greater than or equal to 5.5 years $\left(Y_{i}=\infty\right)$. Patients with observed times less than 5.5 years were either failures or right-censored, and some of those right-censored subjects might indeed have been "cured" patients, but we cannot determine that due to the right-censoring.

We fit the proposed model in (2), where $G(x)$ comes from the family (3) as well as the family (4). We considered values of $\gamma$ in $[0,2]$. The maximum likelihood estimates for the regression coefficients of the proposed class of semiparametric transformation cure models were computed using the proposed method. Furthermore, we selected the best transformation among these two classes as the one that maximized the AIC criterion, which is equivalent to the observed log-likelihood function in this case since the number of parameters is constant. Figure 2 plots the observed log-likelihood functions obtained using the two classes of transformations. Interestingly, both classes select out the same best transformation, which corresponds to the proportional hazards cure model.

Consequently, we report the results from the proportional hazards cure model in the second panel of Table 4. The results show that both interferon treatment and sex did not significantly affect RFS, while age and nodal category did. Younger patients or those with zero positive nodes had significantly better RFS and thus were more likely to be "cured", that is, not to have recurrence of melanoma. The results can also be used to estimate the cure rate for each group. For example, the estimated cure 
rates for a 50 year old female patient with positive nodes under the interferon treatment is $41.0 \%$. Furthermore, we plot in Figure 1 the fitted survival function within each treatment group, where the survival function is calculated as the empirical average of the predicted survival functions within each group. The dashed and dot-dashed lines in Figure 1 present the predicted survival functions and they agree with the Kaplan-Meier curves quite well.

As noted earlier, we treated censored subjects with RFS times 5.5 years or greater as "cured" to estimate the parameters. The choice of such a threshold value can be artificial unless it has some biological meaning. Thus, we also studied the sensitivity of the estimates to the choice of this threshold value. To do this, we varied the threshold value larger than the last failure ( 5 years), using values of $5.1,5.5,6,6.5$ and 7 years. The estimates of the coefficients only differ in the third decimal point, as shown in Table 4.

\section{Discussion}

We have proposed a class of semiparametric transformation cure models which are motivated by a specific biological process. This class is quite broad and it includes the well-known proportional hazards and proportional odds structures as two special cases. We have provided an efficient algorithm for calculating the maximum likelihood estimates. The maximum likelihood estimation procedure yields efficient estimators of the regression parameters. As one by-product, since model (2) reduces to a linear transformation model with a special choice of the link function $\theta(\cdot)$, the algorithm in Section 2 provides a simple way of calculating the maximum likelihood estimates for linear transformation models in general. Specifically, for a linear transformation model with $S\left(t \mid \mathbf{Z}_{i}\right)=G\left\{\exp \left(\boldsymbol{\beta}_{1}^{T} \mathbf{Z}_{i}\right) \Lambda(t)\right\}$, we can reparameterize to make it a cure rate model by defining $F(t)=\Lambda(t) / \Lambda(\tau)$ and adding an intercept term $\log \Lambda(\tau)$ into the regression. Here, $\tau$ refers to the termination time of the study. Thus, treating any subjects censored at time $\tau$ as "cured", we then implement our proposed algorithm to calculate the maximum likelihood estimates of the parameters.

The cure threshold for the E1690 melanoma data was taken to be 5.5 years. The choice of this cutoff value heavily depends on the dataset at hand and other practical elements, including the type of disease, the severity or stage, the corresponding treatment, and other patient prognostic factors that require expert opinion from the physician. A simple guideline is that there should not be any failures after the cure threshold. In fact, the estimates from the proposed method are very robust with respect to the choice of this threshold, as shown in Table 4.

The transformation $G(x)$ can be misspecified in practice due to limited knowledge or complex 
relationships between the covariates and the time-to-event variable. Kosorok et al. (2004) gives some examples in univariate survival data showing that the regression parameters can be estimated up to the correct direction even if $G(x)$ is misspecified. The same ideas can be extended to our proposed model. However, the computation of such estimable quantities in the presence of nonidentifiable parameters is a very challenging problem.

In deriving (2), we assumed that the promotion time survival function, $S^{*}(t)=1-F(t)$, is the same for all tumor cells. One possible generalization to this is to incorporate covariates into $S^{*}(t)$, for example, to allow them to be different across treatments. In this case, the survival function of the tumor cell for the $i$ th subject would be $\exp \left\{-\Lambda(t) e^{\boldsymbol{\zeta}^{T} \mathbf{Z}_{i}}\right\}$, where $\mathbf{Z}_{i}$ is a covariate vector for treatment and other risk factors, and $\mathbf{Z}_{i}$ may share the same components as $\mathbf{X}_{i}$. Thus, the population survival function of interest for subject $i$ is

$$
S\left(t \mid \mathbf{X}_{i}, \mathbf{Z}_{i}\right)=G\left\{\left(1-e^{-\Lambda(t) e} \boldsymbol{\zeta}^{T} \mathbf{z}_{i}\right) \theta\left(\mathbf{X}_{i}\right)\right\}
$$

Issues regarding model identifiability and maximum likelihood estimation in these general models are currently being investigated.

\section{Appendix}

\section{A.1 Proof of Theorem 1}

We introduce the following notation. Let $\mathbf{P}_{n}$ and $\mathbf{P}$ denote the empirical measure of $n$ i.i.d observations and the expectation, respectively; i.e., for any measurable function $g(\Delta, Y, \mathbf{X})$ in $L_{2}(P)$,

$$
\mathbf{P}_{n}[g(\Delta, Y, \mathbf{X})]=\frac{1}{n} \sum_{i=1}^{n} g\left(\Delta_{i}, Y_{i}, \mathbf{X}_{i}\right), \quad \mathbf{P}[g(\Delta, Y, \mathbf{X})]=E[g(\Delta, Y, \mathbf{X})]
$$

From the Lagrange-multiplier calculation, $\hat{F}_{n}$ satisfies the equation that for $Y_{i}<\infty$,

$$
\begin{aligned}
\frac{\Delta_{i}}{F\left\{Y_{i}\right\}}+\sum_{\infty>Y_{j} \geq Y_{i}}\left\{\Delta_{j} \frac{G^{\prime \prime}\left(\eta\left(\hat{\boldsymbol{\beta}}_{n}^{T} \mathbf{X}_{j}\right) F\left(Y_{j}\right)\right) \eta\left(\hat{\boldsymbol{\beta}}_{n}^{T} \mathbf{X}_{j}\right)}{G^{\prime}\left(\eta\left(\hat{\boldsymbol{\beta}}_{n}^{T} \mathbf{X}_{j}\right) F\left(Y_{j}\right)\right)}\right. \\
\left.+\left(1-\Delta_{j}\right) \frac{G^{\prime}\left(\eta\left(\hat{\boldsymbol{\beta}}_{n}^{T} \mathbf{X}_{j}\right) F\left(Y_{j}\right)\right) \eta\left(\hat{\boldsymbol{\beta}}_{n}^{T} \mathbf{X}_{j}\right)}{G\left(\eta\left(\hat{\boldsymbol{\beta}}_{n}^{T} \mathbf{X}_{j}\right) F\left(Y_{j}\right)\right)}\right\}=n \hat{\lambda}_{n} .
\end{aligned}
$$

We multiply both sides by $\hat{F}_{n}\left\{Y_{i}\right\}$ and sum over $Y_{i}$ such that $Y_{i}<\infty$. We get

$$
\hat{\lambda}_{n}=\frac{1}{n} \sum_{i=1}^{n} \Delta_{i} I\left(Y_{i}<\infty\right)+\int_{0}^{\infty} H_{n}\left(y, \hat{\boldsymbol{\beta}}_{n}, \hat{F}_{n}\right) d \hat{F}_{n}(y),
$$


where

$$
\begin{aligned}
H_{n}\left(y, \hat{\boldsymbol{\beta}}_{n}, \hat{F}_{n}\right)=\frac{1}{n}\left[\sum_{Y_{j}<\infty}\right. & \left\{\Delta_{j} \frac{G^{\prime \prime}\left(\eta\left(\hat{\boldsymbol{\beta}}_{n}^{T} \mathbf{X}_{j}\right) \hat{F}_{n}\left(Y_{j}\right)\right) \eta\left(\hat{\boldsymbol{\beta}}_{n}^{T} \mathbf{X}_{j}\right) I\left(Y_{j} \geq y\right)}{G^{\prime}\left(\eta\left(\hat{\boldsymbol{\beta}}_{n}^{T} \mathbf{X}_{j}\right) \hat{F}_{n}\left(Y_{j}\right)\right)}\right. \\
& \left.\left.+\left(1-\Delta_{j}\right) \frac{G^{\prime}\left(\eta\left(\hat{\boldsymbol{\beta}}_{n}^{T} \mathbf{X}_{j}\right) \hat{F}_{n}\left(Y_{j}\right)\right) \eta\left(\hat{\boldsymbol{\beta}}_{n}^{T} \mathbf{X}_{j}\right) I\left(Y_{j} \geq y\right)}{G\left(\eta\left(\hat{\boldsymbol{\beta}}_{n}^{T} \mathbf{X}_{j}\right) \hat{F}_{n}\left(Y_{j}\right)\right)}\right\}\right] .
\end{aligned}
$$

Hence, $\hat{F}_{n}\left\{Y_{i}\right\}=\Delta_{i} / n\left(\hat{\lambda}_{n}-H_{n}\left(Y_{i}, \hat{\boldsymbol{\beta}}_{n}, \hat{F}_{n}\right)\right)$. Obviously, from (A.1.1), $\hat{\lambda}_{n}$ should be bounded by a constant with probability one. Thus, by choosing a subsequence, still indexed by $\{n\}$, we assume $\hat{\lambda}_{n} \rightarrow \lambda^{*}$. By choosing a further subsequence, we assume $\hat{\boldsymbol{\beta}}_{n} \rightarrow \boldsymbol{\beta}^{*}$ and $\hat{F}_{n} \rightarrow F^{*}$ pointwise.

We consider the following class

$$
\mathcal{A}_{1}=\left\{\Delta \frac{G^{\prime \prime}\left(\eta\left(\boldsymbol{\beta}^{T} \mathbf{X}\right) F(Y)\right) \eta\left(\boldsymbol{\beta}^{T} \mathbf{X}\right) I(\infty>Y \geq y)}{G^{\prime}\left(\eta\left(\boldsymbol{\beta}^{T} \mathbf{X}\right) F(Y)\right)}+(1-\Delta) \frac{G^{\prime}\left(\eta\left(\boldsymbol{\beta}^{T} \mathbf{X}\right) F(Y)\right) \eta\left(\boldsymbol{\beta}^{T} \mathbf{X}\right) I(\infty>Y \geq y)}{G\left(\eta\left(\boldsymbol{\beta}^{T} \mathbf{X}\right) F(Y)\right)}:\right.
$$

$F$ is a distribution function, $\left.\boldsymbol{\beta} \in \mathcal{B}_{0}, y \in[0, \infty)\right\}$.

First, $\left\{\boldsymbol{\beta}^{T} \mathbf{X}: \boldsymbol{\beta} \in \mathcal{B}_{0}\right\}$ and $\{F(Y): F$ is a distribution function $\}$ are both Donsker classes, where the latter follows from Theorem 2.7.5 of van der Vaart and Wellner (1996). Since $G, G^{\prime}, G^{\prime \prime}$ and $\eta$ are continuously differentiable functions, the preservation of the Donsker property based on Theorem 2.10.6 of van der Vaart and Wellner (1996) implies that the classes

$$
\left\{G^{(k)}\left(\eta\left(\boldsymbol{\beta}^{T} \mathbf{X}\right) F(Y)\right): \boldsymbol{\beta} \in \mathcal{B}_{0}, F \text { is a distribution function }\right\}, \quad k=0,1,2,
$$

and $\left\{\eta\left(\boldsymbol{\beta}^{T} \mathbf{X}\right): \boldsymbol{\beta} \in \mathcal{B}_{0}\right\}$ are Donsker classes. Furthermore, we note $G^{\prime}(x)$ and $G(x)$ are both bounded away from zero when $x$ is in a compact set. Thus, the preservation of the Donsker property under the summation, product and quotient, as given in Examples 2.10.7-2.10.9 of van der Vaart and Wellner (1996) gives that the class $\mathcal{A}_{1}$ is a Donsker class so is also a Glivenko-Cantelli class. As a result of the Glivenko-Cantelli theorem and the bounded convergence theorem, we conclude that uniformly in $y, H_{n}\left(y, \hat{\boldsymbol{\beta}}_{n}, \hat{F}_{n}\right) \rightarrow H^{*}(y)$, where

$$
\begin{aligned}
& H^{*}(y)=E\left[\Delta \frac{G^{\prime \prime}\left(\eta\left(\boldsymbol{\beta}^{* T} \mathbf{X}\right) F^{*}(Y)\right) \eta\left(\boldsymbol{\beta}^{* T} \mathbf{X}\right) I(\infty>Y \geq y)}{G^{\prime}\left(\eta\left(\boldsymbol{\beta}^{* T} \mathbf{X}\right) F^{*}(Y)\right)}\right. \\
&\left.+(1-\Delta) \frac{G^{\prime}\left(\eta\left(\boldsymbol{\beta}^{* T} \mathbf{X}\right) F^{*}(Y)\right) \eta\left(\boldsymbol{\beta}^{* T} \mathbf{X}\right) I(\infty>Y \geq y)}{G\left(\eta\left(\boldsymbol{\beta}^{* T} \mathbf{X}\right) F^{*}(Y)\right)}\right] .
\end{aligned}
$$

Moreover, the right-hand side of (A.1.1) converges to

$$
\lambda^{*}=E\{\Delta I(Y<\infty)\}+E\left\{I(Y<\infty) \int_{0}^{Y} H^{*}(y) d F^{*}(y)\right\} .
$$


Now we wish to show that $\left|\lambda^{*}-H^{*}(y)\right|>\delta^{*}$ for some positive constant $\delta^{*}$. To see that, we first note that from $\sum_{i=1}^{n} \hat{F}_{n}\left\{Y_{i}\right\}=1$, then

$$
\begin{aligned}
1=\sum_{i=1}^{n} I\left(Y_{i}<\infty\right) & \frac{\Delta_{i}}{n\left(\hat{\lambda}_{n}-H_{n}\left(Y_{i}, \hat{\boldsymbol{\beta}}_{n}, \hat{F}_{n}\right)\right)}=\sum_{i=1}^{n} I\left(Y_{i}<\infty\right) \frac{\Delta_{i}}{n\left|\hat{\lambda}_{n}-H_{n}\left(Y_{i}, \hat{\boldsymbol{\beta}}_{n}, \hat{F}_{n}\right)\right|} \\
& \geq \frac{1}{n} \sum_{i=1}^{n} I\left(Y_{i}<\infty\right) \frac{\Delta_{i}}{\left|\hat{\lambda}_{n}-H_{n}\left(Y_{i}, \hat{\boldsymbol{\beta}}_{n}, \hat{F}_{n}\right)\right|+\epsilon},
\end{aligned}
$$

for any positive constant $\epsilon$. Since $H_{n}\left(y, \hat{\boldsymbol{\beta}}_{n}, \hat{F}_{n}\right)$ converges uniformly to $H^{*}(y)$,

$$
\frac{1}{n} \sum_{i=1}^{n} I\left(Y_{i}<\infty\right) \frac{\Delta_{i}}{\left|\hat{\lambda}_{n}-H_{n}\left(Y_{i}, \hat{\boldsymbol{\beta}}_{n}, \hat{F}_{n}\right)\right|+\epsilon}-\frac{1}{n} \sum_{i=1}^{n} I\left(Y_{i}<\infty\right) \frac{\Delta_{i}}{\left|\lambda^{*}-H^{*}\left(Y_{i}\right)\right|+\epsilon} \rightarrow 0 .
$$

Then after taking limits on both sides, we obtain $1 \geq E\left\{\Delta I(Y<\infty) /\left(\left|\lambda^{*}-H^{*}(Y)\right|+\epsilon\right)\right\}$. Letting $\epsilon \rightarrow 0$, by the monotone convergence theorem, we have

$$
1 \geq \int_{0}^{\infty} \frac{c_{0} d y}{\left|\lambda^{*}-H^{*}(y)\right|}
$$

where $c_{0}$ is a positive constant. Thus, if $\inf _{y}\left|\lambda^{*}-H^{*}(y)\right|=0$, we claim that there exists a finite $y_{0}$ such that $H^{*}\left(y_{0}\right)=\lambda^{*}$. Otherwise, $H^{*}(\infty)=\lambda^{*}=0$. Then, for large $y,\left|\lambda^{*}-H^{*}(y)\right|<1$ which makes (A.1.3) impossible. Now suppose that there exists a finite $y_{0}$ such that $\lambda^{*}=H^{*}\left(y_{0}\right)$. Then (A.1.3) becomes $1 \geq c_{0} \int_{0}^{\infty} d y /\left|H^{*}\left(y_{0}\right)-H^{*}(y)\right|$. This is impossible since $H^{*}(y)$ is continuously differentiable in a neighborhood of $y_{0}$. Therefore, there exists a positive constant $\delta^{*}$ such that $\left|\lambda^{*}-H^{*}(y)\right|>\delta^{*}$. This implies that when $n$ is large, $\left|\hat{\lambda}_{n}-H_{n}\left(y, \hat{\boldsymbol{\beta}}_{n}, \hat{F}_{n}\right)\right|>\delta^{*}$. Note that $\hat{F}_{n}(y)=n^{-1} \sum_{i=1}^{n} \Delta_{i} I\left(Y_{i} \leq y\right) /\left|\hat{\lambda}_{n}-H_{n}\left(Y_{i}, \hat{\boldsymbol{\beta}}_{n}, \hat{F}_{n}\right)\right|$ so $\hat{F}_{n}(y)$ converges uniformly to $F^{*}(y)=$ $E\left\{\Delta I(Y \leq y) /\left|\lambda^{*}-H^{*}(Y)\right|\right\}$.

We now show that $\boldsymbol{\beta}^{*}=\boldsymbol{\beta}_{0}, F^{*}=F_{0}$. To do so, we construct another function $\tilde{F}$ which has jumps only at $Y_{i}$ such that $\Delta_{i}=1$ and $Y_{i}<\infty$. Moreover,

$$
\tilde{F}_{n}\left\{Y_{i}\right\}=\frac{1}{n c_{n}} \frac{\Delta_{i}}{\tilde{\lambda}_{n}-H_{n}\left(Y_{i}, \boldsymbol{\beta}_{0}, F_{0}\right)},
$$

where $\tilde{\lambda}_{n}$ satisfies a similar equation to (A.1.1) and it is given by

$$
\tilde{\lambda}_{n}=\frac{1}{n} \sum_{i=1}^{n} \Delta_{i} I\left(Y_{i}<\infty\right)+\int_{0}^{\infty} H_{n}\left(y, \boldsymbol{\beta}_{0}, F_{0}\right) d F_{0}(y)
$$

and $c_{n}$ is a constant such that $\sum_{i=1}^{n} \tilde{F}_{n}\left\{Y_{i}\right\}=1$. Furthermore, using the argument of the GlivenkoCantelli property as before, we can easily show that uniformly in $y, H_{n}\left(y, \boldsymbol{\beta}_{0}, F_{0}\right)$ converges to

$$
\tilde{H}(y)=E\left\{\Delta \frac{G^{\prime \prime}\left(\eta\left(\boldsymbol{\beta}_{0}^{T} \mathbf{X}\right) F_{0}(Y)\right) \eta\left(\boldsymbol{\beta}_{0}^{T} \mathbf{X}\right) I(\infty>Y \geq y)}{G^{\prime}\left(\eta\left(\boldsymbol{\beta}_{0}^{T} \mathbf{X}\right) F_{0}(Y)\right)}\right.
$$




$$
\left.+(1-\Delta) \frac{G^{\prime}\left(\eta\left(\boldsymbol{\beta}_{0}^{T} \mathbf{X}\right) F_{0}(Y)\right) \eta\left(\boldsymbol{\beta}_{0}^{T} \mathbf{X}\right) I(\infty>Y \geq y)}{G\left(\eta\left(\boldsymbol{\beta}_{0}^{T} \mathbf{X}\right) F_{0}(Y)\right)}\right\}
$$

which, after integration by part, is equal to $E\left[\eta\left(\boldsymbol{\beta}_{0}^{T} \mathbf{X}\right) G^{\prime}\left(\eta\left(\boldsymbol{\beta}_{0}^{T} \mathbf{X}\right) F_{0}(y)\right) S_{c}(y \mid \mathbf{X})\right]$, where $S_{c}$ is the conditional survival function of the censoring time. Consequently, direct calculation gives that $\tilde{\lambda}_{n}$ converges to 0. Furthermore, from equation

$$
c_{n} \tilde{F}_{n}(y)=\frac{1}{n} \sum_{i=1}^{n} \frac{\Delta_{i} I\left(Y_{i} \leq y\right)}{\tilde{\lambda}_{n}-H_{n}\left(Y_{i}, \boldsymbol{\beta}_{0}, F_{0}\right)},
$$

we obtain that uniformly in $y, c_{n} \tilde{F}_{n}(y)$ converges to

$$
E\left[\frac{\Delta I(Y \leq y)}{-\left.E\left\{S_{c}(\tilde{y} \mid \mathbf{X}) \eta\left(\boldsymbol{\beta}_{0}^{T} \mathbf{X}\right) G^{\prime}\left(\eta\left(\boldsymbol{\beta}_{0}^{T} \mathbf{X}\right) F_{0}(\tilde{y})\right)\right\}\right|_{\tilde{y}=Y}}\right]=F_{0}(y) .
$$

Hence, $c_{n} \rightarrow 1$ and $\tilde{F}_{n}(y)$ converges to $F_{0}(y)$ uniformly.

Note that $\hat{F}_{n}$ is absolutely continuous with respect to $\tilde{F}_{n}(y)$ with

$$
\hat{F}_{n}(y)=\int_{0}^{y} \frac{\left|\tilde{\lambda}_{n}-\tilde{H}_{n}\left(t, \boldsymbol{\beta}_{0}, F_{0}\right)\right|}{\left|\hat{\lambda}_{n}-H_{n}\left(t, \hat{\boldsymbol{\beta}}_{n}, \hat{F}_{n}\right)\right|} d \tilde{F}_{n}(t) .
$$

From the forgoing arguments, the integrand in (A.1.4) is bounded and uniformly converges to $|\tilde{H}(t)|$ $/\left|\lambda^{*}-H^{*}(t)\right|$. We conclude that $F^{*}(y)=\int_{0}^{y}|\tilde{H}(t)| d F_{0}(t) /\left|\lambda^{*}-H^{*}(t)\right|$. This implies that $F^{*}$ is absolutely continuous with respect to $F_{0}$. Therefore, $F^{*}$ is also differentiable and we denote its density function by $f^{*}$.

On the other hand, since the observed log-likelihood function at $\left(\hat{\boldsymbol{\beta}}_{n}, \hat{F}_{n}\right)$ is larger than or equal to the observed log-likelihood function at $\left(\boldsymbol{\beta}_{0}, \tilde{F}_{n}\right)$, we have

$$
\begin{aligned}
& \frac{1}{n} \sum_{i=1}^{n} I\left(Y_{i}<\infty\right) \Delta_{i} \log \frac{\hat{F}_{n}\left\{Y_{i}\right\}}{\tilde{F}_{n}\left\{Y_{i}\right\}}+\frac{1}{n} \sum_{i=1}^{n}\left\{I\left(Y_{i}=\infty\right) \log \frac{G\left(\eta\left(\hat{\boldsymbol{\beta}}_{n}^{T} \mathbf{X}_{i}\right)\right)}{G\left(\eta\left(\boldsymbol{\beta}_{0}^{T} \mathbf{X}_{i}\right)\right)}\right\} \\
& \quad+\frac{1}{n} \sum_{i=1}^{n} I\left(Y_{i}<\infty\right)\left\{\Delta_{i} \log \frac{G^{\prime}\left(\eta\left(\hat{\boldsymbol{\beta}}_{n}^{T} \mathbf{X}_{i}\right) \hat{F}_{n}\left(Y_{i}\right)\right) \eta\left(\hat{\boldsymbol{\beta}}_{n}^{T} \mathbf{X}_{i}\right)}{G^{\prime}\left(\eta\left(\boldsymbol{\beta}_{0}^{T} \mathbf{X}_{i}\right) \tilde{F}_{n}\left(Y_{i}\right)\right) \eta\left(\boldsymbol{\beta}_{0}^{T} \mathbf{X}_{i}\right)}+\left(1-\Delta_{i}\right) \log \frac{G\left(\eta\left(\hat{\boldsymbol{\beta}}_{n}^{T} \mathbf{X}_{i}\right) \hat{F}_{n}\left(Y_{i}\right)\right)}{G\left(\eta\left(\boldsymbol{\beta}_{0}^{T} \mathbf{X}_{i}\right) \tilde{F}_{n}\left(Y_{i}\right)\right)}\right\} \geq 0 .
\end{aligned}
$$

We take limits on both sides and note that

$$
\frac{1}{n} \sum_{i=1}^{n} \Delta_{i} I\left(Y_{i}<\infty\right) \log \frac{\hat{F}_{n}\left\{Y_{i}\right\}}{\tilde{F}_{n}\left\{Y_{i}\right\}} \rightarrow E\left\{\Delta I(Y<\infty) \log \frac{f^{*}(Y)}{f_{0}(Y)}\right\} .
$$

We obtain $-K\left(\left(\boldsymbol{\beta}^{*}, F^{*}\right),\left(\boldsymbol{\beta}_{0}, F_{0}\right)\right) \geq 0$, where $K(\cdot, \cdot)$ denotes the Kullback-Leibler information of $\left(\boldsymbol{\beta}^{*}, F^{*}\right)$ with respect to the true parameters. Immediately, we obtain

$$
\begin{aligned}
& \left\{-G^{\prime}\left(\eta\left(\boldsymbol{\beta}^{* T} \mathbf{X}\right) F^{*}(Y)\right) \eta\left(\boldsymbol{\beta}^{* T} \mathbf{X}\right) f^{*}(Y)\right\}^{\Delta I(Y<\infty)}\left\{G\left(\eta\left(\boldsymbol{\beta}^{* T} \mathbf{X}\right) F^{*}(Y)\right)\right\}^{(1-\Delta) I(Y<\infty)+I(Y=\infty)} \\
= & \left\{-G^{\prime}\left(\eta\left(\boldsymbol{\beta}_{0}^{T} \mathbf{X}\right) F_{0}(Y)\right) \eta\left(\boldsymbol{\beta}_{0}^{T} \mathbf{X}\right) f_{0}(Y)\right\}^{\Delta I(Y<\infty)}\left\{G\left(\eta\left(\boldsymbol{\beta}_{0}^{T} \mathbf{X}\right) F_{0}(Y)\right)\right\}^{(1-\Delta) I(Y<\infty)+I(Y=\infty)}
\end{aligned}
$$


for almost every $(\Delta, X, Y)$ in its support. According to the second paragraph in Section 3, we obtain $\boldsymbol{\beta}^{*}=\boldsymbol{\beta}_{0}$ and $F^{*}=F_{0}$.

We have shown that for almost every sample in the probability space, we can always choose a subsequence of $\left(\hat{\boldsymbol{\beta}}_{n}, \hat{F}_{n}\right)$ so that it converges to $\left(\boldsymbol{\beta}_{0}, F_{0}\right)$. Hence, with probability one, $\hat{\boldsymbol{\beta}}_{n} \rightarrow \boldsymbol{\beta}_{0}$ and $\hat{F}_{n}(y) \rightarrow F_{0}(y)$ for every $y \in[0, \infty)$. Particularly, we obtain $\sup _{y}\left|\hat{F}_{n}(y)-F_{0}(y)\right| \rightarrow 0$ due to the continuity of $F_{0}$.

Remark A.1 When transformation $G$ depends on some unknown parameter $\gamma$, where $\gamma$ belongs to a compact set $\Gamma$, the proof of the consistency applies when assumptions $(\mathrm{C} 1)$ and $(\mathrm{C} 3)$ are replaced by (C1'). Parameters $\left(\boldsymbol{\beta}_{0}, \gamma_{0}, F_{0}\right)$ are identifiable.

$\left(\mathrm{C} 3^{\prime}\right) . G_{\gamma}(x)$ is three times differentiable with respect to $\gamma$ and $x$ and all the derivatives are uniformly bounded with $G_{\gamma}^{\prime}(x)>0$.

Especially, (C3') ensures the classes of random functions in the above proof to be the Glivenko-Cantelli classes while $\left(\mathrm{C} 1^{\prime}\right)$ ensures the limit of $\left(\hat{\boldsymbol{\beta}}_{n}, \hat{\gamma}_{n}, \hat{F}_{n}\right)$ must be the true parameters.

\section{A.2 Proof of Theorem 2}

To prove the asymptotic properties of $\left(\hat{\boldsymbol{\beta}}_{n}, \hat{F}_{n}\right)$, we recall the definition of $\mathcal{H}$ in Section 3 . Furthermore, we abbreviate $l(\boldsymbol{\beta}, F)$ as the $\log$-likelihood function of (5), given by

$$
\begin{aligned}
l(\boldsymbol{\beta}, F)= & I(Y<\infty)\left[\Delta \log f+\Delta \log \left\{-G^{\prime}\left(\eta\left(\boldsymbol{\beta}^{T} \mathbf{X}\right) F(Y)\right) \eta\left(\boldsymbol{\beta}^{T} \mathbf{X}\right)\right\}\right. \\
& \left.+(1-\Delta) \log G\left(\eta\left(\boldsymbol{\beta}^{T} \mathbf{X}\right) F(Y)\right)\right]+I(Y=\infty) \log G\left(\eta\left(\boldsymbol{\beta}^{T} \mathbf{X}\right)\right) .
\end{aligned}
$$

Denote $l_{\boldsymbol{\beta}}(\boldsymbol{\beta}, F)$ as the derivative of $l(\boldsymbol{\beta}, F)$ with respect to $\boldsymbol{\beta}$ and denote $l_{F}(\boldsymbol{\beta}, F)\left[\int\left(h_{2}-Q_{F}\left[h_{2}\right]\right) d F\right]$ as the derivative of $l(\boldsymbol{\beta}, F)$ along the path $\left(\boldsymbol{\beta}, F_{\epsilon}=F+\epsilon \int Q_{F}\left(h_{2}\right) d F\right), \epsilon \in\left(-\epsilon_{0}, \epsilon_{0}\right)$ for a small constant $\epsilon_{0}$, where $Q_{F}\left[h_{2}\right]=h_{2}(t)-\int_{0}^{\infty} h_{2}(t) d F(t)$. Additionally, we can define the derivative of $l_{\boldsymbol{\beta}}(\boldsymbol{\beta}, F)$ with respect to $\boldsymbol{\beta}$, denoted by $l_{\boldsymbol{\beta} \boldsymbol{\beta}}(\boldsymbol{\beta}, F)$, the derivative of $l_{\boldsymbol{\beta}}(\boldsymbol{\beta}, F)$ with respect to $F$ along the path $F+\epsilon\left(\hat{F}_{n}-F\right)$, denoted by $l_{\boldsymbol{\beta}_{F}}\left[\hat{F}_{n}-F\right]$, and the derivative of $l_{F}(\boldsymbol{\beta}, F)\left[\int Q_{F}\left(h_{2}\right) d F\right]$ with respect to $\boldsymbol{\beta}$, denoted by $l_{F \boldsymbol{\beta}}(\boldsymbol{\beta}, F)\left[\int Q_{F}\left(h_{2}\right) d F\right]$, the derivative $l_{F}(\boldsymbol{\beta}, F)\left[\int Q_{F}\left(h_{2}\right) d F\right]$ with respect to $F$ along the path $F+\epsilon\left(\hat{F}_{n}-F\right)$, denoted by $l_{F F}(\boldsymbol{\beta}, F)\left[\int Q_{F}\left(h_{2}\right) d F, \hat{F}_{n}-F\right]$. Furthermore, we define

$$
\begin{aligned}
\Psi_{1}(\Delta, Y, \mathbf{X})= & I(Y<\infty) \Delta\left\{\frac{G^{(3)}\left(\eta\left(\boldsymbol{\beta}^{T} \mathbf{X}\right) F(Y)\right)}{G^{\prime}\left(\eta\left(\boldsymbol{\beta}^{T} \mathbf{X}\right) F(Y)\right)}-\frac{G^{\prime \prime}\left(\eta\left(\boldsymbol{\beta}^{T} \mathbf{X}\right) F(Y)\right)^{2}}{G^{\prime}\left(\eta\left(\boldsymbol{\beta}^{T} \mathbf{X}\right) F(Y)\right)^{2}}\right\} \\
& +\{(1-\Delta) I(Y<\infty)+I(Y=\infty)\} \frac{G^{\prime \prime}\left(\eta\left(\boldsymbol{\beta}^{T} \mathbf{X}\right) F(Y)\right)}{G\left(\eta\left(\boldsymbol{\beta}^{T} \mathbf{X}\right) F(Y)\right)} \\
& -\{(1-\Delta) I(Y<\infty)+I(Y=\infty)\} \frac{G^{\prime}\left(\eta\left(\boldsymbol{\beta}^{T} \mathbf{X}\right) F(Y)\right)^{2}}{G\left(\eta\left(\boldsymbol{\beta}^{T} \mathbf{X}\right) F(Y)\right)^{2}},
\end{aligned}
$$




$$
\begin{aligned}
\Psi_{2}(\Delta, Y, \mathbf{X})= & I(Y<\infty) \Delta \frac{G^{\prime \prime}\left(\eta\left(\boldsymbol{\beta}^{T} \mathbf{X}\right) F(Y)\right)}{G^{\prime}\left(\eta\left(\boldsymbol{\beta}^{T} \mathbf{X}\right) F(Y)\right)} \\
& +\{(1-\Delta) I(Y<\infty)+I(Y=\infty)\} \frac{G^{\prime}\left(\eta\left(\boldsymbol{\beta}^{T} \mathbf{X}\right) F(Y)\right)}{G\left(\eta\left(\boldsymbol{\beta}^{T} \mathbf{X}\right) F(Y)\right)}
\end{aligned}
$$

Since $\left(\hat{\boldsymbol{\beta}}_{n}, \hat{F}_{n}\right)$ maximizes $\mathbf{P}_{n} l(\boldsymbol{\beta}, F)$, for any $\left(\mathbf{h}_{1}, h_{2}\right) \in \mathcal{H}$, it follows that

$$
\mathbf{P}_{n}\left\{l_{\boldsymbol{\beta}}\left(\hat{\boldsymbol{\beta}}_{n}, \hat{F}_{n}\right)^{T} \mathbf{h}_{1}+l_{F}\left(\hat{\boldsymbol{\beta}}_{n}, \hat{F}_{n}\right)\left[\int Q_{\hat{F}_{n}}\left(h_{2}\right) d \hat{F}_{n}\right]\right\}=0 .
$$

Note that $\mathbf{P}\left\{l_{\boldsymbol{\beta}}\left(\boldsymbol{\beta}_{0}, F_{0}\right)^{T} \mathbf{h}_{1}+l_{F}\left(\boldsymbol{\beta}_{0}, F_{0}\right)\left[\int Q_{F_{0}}\left(h_{2}\right) d F_{0}\right]\right\}=0$. Thus, we obtain

$$
\begin{aligned}
\sqrt{n}\left(\mathbf{P}_{n}-\mathbf{P}\right)\left\{l_{\boldsymbol{\beta}}\left(\hat{\boldsymbol{\beta}}_{n}, \hat{F}_{n}\right)^{T} \mathbf{h}_{1}+l_{F}\left(\hat{\boldsymbol{\beta}}_{n}, \hat{F}_{n}\right)\left[\int Q_{\hat{F}_{n}}\left(h_{2}\right) d \hat{F}_{n}\right]\right\} \\
=-\sqrt{n} \mathbf{P}\left\{l_{\boldsymbol{\beta}}\left(\hat{\boldsymbol{\beta}}_{n}, \hat{F}_{n}\right)^{T} \mathbf{h}_{1}+l_{F}\left(\hat{\boldsymbol{\beta}}_{n}, \hat{F}_{n}\right)\left[\int Q_{\hat{F}_{n}}\left(h_{2}\right) d \hat{F}_{n}\right]\right\} \\
+\sqrt{n} \mathbf{P}\left\{l_{\boldsymbol{\beta}}\left(\boldsymbol{\beta}_{0}, F_{0}\right)^{T} \mathbf{h}_{1}+l_{F}\left(\boldsymbol{\beta}_{0}, F_{0}\right)\left[\int Q_{F_{0}}\left(h_{2}\right) d F_{0}\right]\right\} .
\end{aligned}
$$

First, by the same arguments in the consistency proof, the classes of

$$
\begin{aligned}
& \mathcal{A}_{2}=\left\{\frac{G^{\prime}(x)}{G(x)},\left.\frac{G^{\prime \prime}(x)}{G^{\prime}(x)}\right|_{x=\eta\left(\boldsymbol{\beta}^{T} \mathbf{X}\right) F(Y)}:\left\|\boldsymbol{\beta}-\boldsymbol{\beta}_{0}\right\|<\delta_{0}, \sup _{y}\left|F(y)-F_{0}(y)\right|<\delta_{0}\right\}, \\
& \mathcal{A}_{3}=\left\{\eta^{\prime}\left(\boldsymbol{\beta}^{T} \mathbf{X}\right) F(Y), \eta\left(\boldsymbol{\beta}^{T} \mathbf{X}\right) F(Y):\left\|\boldsymbol{\beta}-\boldsymbol{\beta}_{0}\right\|<\delta_{0}, \sup _{y}\left|F(y)-F_{0}(y)\right|<\delta_{0}\right\}
\end{aligned}
$$

are P-Donsker. Additionally, it is clear to see both classes $\left\{Q_{F}\left(h_{2}\right):\left\|h_{2}\right\|_{V} \leq 1, \sup _{y}\left|F(y)-F_{0}(y)\right|<\delta_{0}\right\}$ and $\left\{\int_{0}^{Y} Q_{F}\left(h_{2}\right) d F:\left\|h_{2}\right\|_{V} \leq 1, \sup _{y}\left|F(y)-F_{0}(y)\right|<\delta_{0}\right\}$ contain the functions of $Y$ with bounded variations so they are also P-Donsker. Therefore, from the explicit expression of $l_{\boldsymbol{\beta}}$ and $l_{F}$, the preservation of the Donsker classes under algebraic operations implies that the class

$$
\mathcal{A}_{4}=\left\{l_{\boldsymbol{\beta}}(\boldsymbol{\beta}, F)^{T} \mathbf{h}_{1}+l_{F}(\boldsymbol{\beta}, F)\left[\int Q_{F}\left(h_{2}\right) d F\right]:\left\|\mathbf{h}_{1}\right\| \leq 1,\left\|h_{2}\right\|_{V} \leq 1,\left\|\boldsymbol{\beta}-\boldsymbol{\beta}_{0}\right\|+\sup _{y}\left|F(y)-F_{0}(y)\right|<\delta_{0}\right\}
$$

is P-Donsker. On the other hand, it is straightforward to show that

$$
l_{\boldsymbol{\beta}}\left(\hat{\boldsymbol{\beta}}_{n}, \hat{F}_{n}\right)^{T} \mathbf{h}_{1}+l_{F}\left(\hat{\boldsymbol{\beta}}_{n}, \hat{F}_{n}\right)\left[\int Q_{\hat{F}_{n}}\left(h_{2}\right) d \hat{F}_{n}\right] \rightarrow l_{\boldsymbol{\beta}}\left(\boldsymbol{\beta}_{0}, F_{0}\right)^{T} \mathbf{h}_{1}+l_{F}\left(\boldsymbol{\beta}_{0}, F_{0}\right)\left[\int Q_{F_{0}}\left(h_{2}\right) d F_{0}\right]
$$

uniformly in $\left(\mathbf{h}_{1}, h_{2}\right) \in \mathcal{H}$. Thus, the left-hand side of (A.2.1) is equal to

$$
\sqrt{n}\left(\mathbf{P}_{n}-\mathbf{P}\right)\left\{l_{\boldsymbol{\beta}}\left(\boldsymbol{\beta}_{0}, F_{0}\right)^{T} \mathbf{h}_{1}+l_{F}\left(\boldsymbol{\beta}_{0}, F_{0}\right)\left[\int Q_{F_{0}}\left(h_{2}\right) d F_{0}\right]\right\}+o_{p}(1)
$$

where $o_{p}(1)$ is a random variable that converges to zero in probability in the metric space $l^{\infty}(\mathcal{H})$. As a result, the left-hand side of (A.2.1) converges weakly to a zero-mean Gaussian process in $l^{\infty}(\mathcal{H})$. 
Second, simple algebra shows that uniformly in $\left(\mathbf{h}_{1}, h_{2}\right) \in \mathcal{H}$,

$$
\begin{gathered}
\mid l_{\boldsymbol{\beta}}\left(\hat{\boldsymbol{\beta}}_{n}, \hat{F}_{n}\right)^{T} \mathbf{h}_{1}+l_{F}\left(\hat{\boldsymbol{\beta}}_{n}, \hat{F}_{n}\right)\left[\int Q_{\hat{F}_{n}}\left(h_{2}\right) d \hat{F}_{n}\right]-l_{\boldsymbol{\beta}}\left(\boldsymbol{\beta}_{0}, F_{0}\right)^{T} \mathbf{h}_{1}-l_{F}\left(\boldsymbol{\beta}_{0}, F_{0}\right)\left[\int Q_{F_{0}}\left(h_{2}\right) d F_{0}\right] \\
-\left\{\left(\hat{\boldsymbol{\beta}}_{n}-\boldsymbol{\beta}_{0}\right)^{T} l_{\boldsymbol{\beta} \boldsymbol{\beta}}\left(\boldsymbol{\beta}_{0}, F_{0}\right) \mathbf{h}_{1}+\left(\hat{\boldsymbol{\beta}}_{n}-\boldsymbol{\beta}_{0}\right)^{T} l_{F \boldsymbol{\beta}}\left(\boldsymbol{\beta}_{0}, F_{0}\right)\left[\int Q_{F_{0}}\left(h_{2}\right) d F_{0}\right]\right. \\
\left.\quad+\mathbf{h}_{1}^{T} l_{\boldsymbol{\beta}_{F}}\left[\hat{F}_{n}-F_{0}\right]+l_{F F}\left(\boldsymbol{\beta}_{0}, F_{0}\right)\left[\int Q_{F_{0}}\left(h_{2}\right) d F_{0}, \hat{F}_{n}-F_{0}\right]\right\} \mid \\
\leq o_{p}\left\{\left\|\hat{\boldsymbol{\beta}}_{n}-\boldsymbol{\beta}_{0}\right\|+\left\|\hat{F}_{n}-F_{0}\right\|_{l^{\infty}}\right\} .
\end{gathered}
$$

Thus, combining with the expressions of $l_{\boldsymbol{\beta} \boldsymbol{\beta}}, l_{\boldsymbol{\beta} F}, l_{F \boldsymbol{\beta}}$ and $l_{F F}$, we obtain that the right-hand side of (A.2.1) equals

$$
\begin{gathered}
-\sqrt{n}\left\{\left(\hat{\boldsymbol{\beta}}_{n}-\boldsymbol{\beta}_{0}\right)^{T} \Omega_{\boldsymbol{\beta}}\left(\mathbf{h}_{1}, Q_{F_{0}}\left(h_{2}\right)\right)+\int_{0}^{\infty} \Omega_{F}\left(\mathbf{h}_{1}, Q_{F_{0}}\left(h_{2}\right)\right) d\left(\hat{F}_{n}-F_{0}\right)(y)\right\} \\
+o\left\{\sqrt{n}\left(\left\|\hat{\boldsymbol{\beta}}_{n}-\boldsymbol{\beta}_{0}\right\|+\left\|\hat{F}_{n}-F_{0}\right\|_{l \infty}\right)\right\},
\end{gathered}
$$

where

$$
\begin{aligned}
& \Omega_{\boldsymbol{\beta}}\left(\mathbf{h}_{1}, Q_{F_{0}}\left(h_{2}\right)\right)=E\left[I(Y<\infty) \Delta \frac{\eta^{\prime \prime}\left(\boldsymbol{\beta}^{T} \mathbf{X}\right) \eta\left(\boldsymbol{\beta}^{T} \mathbf{X}\right)-\eta^{\prime}\left(\boldsymbol{\beta}^{T} \mathbf{X}\right)^{2}}{\eta\left(\boldsymbol{\beta}^{T} \mathbf{X}\right)^{2}} \mathbf{X X}^{T} \mathbf{h}_{1}\right] \\
& +E\left[\left\{\Psi_{1}^{0}(\Delta, Y, \mathbf{X}) \eta^{\prime}\left(\boldsymbol{\beta}_{0}^{T} \mathbf{X}\right)^{2} F_{0}(Y)^{2}+\Psi_{2}^{0}(\Delta, Y, \mathbf{X}) \eta^{\prime \prime}\left(\boldsymbol{\beta}_{0}^{T} \mathbf{X}\right) F_{0}(Y)\right\} \mathbf{X X}^{T} \mathbf{h}_{1}\right] \\
& +E\left[\left\{\Psi_{1}^{0}(\Delta, Y, \mathbf{X}) \eta\left(\boldsymbol{\beta}_{0}^{T} \mathbf{X}\right) \eta^{\prime}\left(\boldsymbol{\beta}_{0}^{T} \mathbf{X}\right) F_{0}(Y)+\Psi_{2}^{0}(\Delta, Y, \mathbf{X}) \eta^{\prime}\left(\boldsymbol{\beta}_{0}^{T} \mathbf{X}\right) F_{0}(Y)\right\} \mathbf{X}\right. \\
& \left.\times \int_{0}^{Y} Q_{F_{0}}\left(h_{2}\right) d F_{0}\right] \\
& \Omega_{F}\left(\mathbf{h}_{1}, Q_{F_{0}}\left(h_{2}\right)\right)=-E\left[I(Y<\infty) \Delta+\Psi_{2}^{0}(\Delta, Y, \mathbf{X}) \eta\left(\boldsymbol{\beta}_{0}^{T} \mathbf{X}\right)\left\{F_{0}(Y)-I(Y \geq y)\right\}\right] Q_{F_{0}}\left[h_{2}\right] \\
& +E\left[\left\{\Psi_{1}^{0}(\Delta, Y, \mathbf{X}) \eta\left(\boldsymbol{\beta}_{0}^{T} \mathbf{X}\right) \eta^{\prime}\left(\boldsymbol{\beta}_{0}^{T} \mathbf{X}\right) F_{0}(Y)+\Psi_{2}^{0}(\Delta, Y, \mathbf{X}) \eta^{\prime}\left(\boldsymbol{\beta}_{0}^{T} \mathbf{X}\right) F_{0}(Y)\right\}\right. \\
& \left.\times \mathbf{X}^{T} \mathbf{h}_{1} I(Y \geq y)\right] \\
& +E\left[I(Y \geq y) \Psi_{1}^{0}(\Delta, Y, \mathbf{X}) \eta\left(\boldsymbol{\beta}_{0}^{T} \mathbf{X}\right)^{2} \int_{0}^{Y} Q_{F_{0}}\left(h_{2}\right) d F_{0}\right],
\end{aligned}
$$

where $\Psi_{1}^{0}$ and $\Psi_{2}^{0}$ have the same expressions as $\Psi_{1}$ and $\Psi_{2}$ respectively but with $\boldsymbol{\beta}$ and $F$ replaced by $\boldsymbol{\beta}_{0}$ and $F_{0}$.

Third, the linear operator $\left(\Omega_{\boldsymbol{\beta}}, \Omega_{F}\right)$ is a bounded linear operator from the linear space

$$
\mathcal{S}=R^{d} \times\left\{\tilde{h}_{2}:\left\|\tilde{h}_{2}\right\|_{V}<\infty, \int_{0}^{\infty} \tilde{h}_{2}(y) d F_{0}(y)=0\right\}
$$

to itself. We wish to show that $\left(\Omega_{\boldsymbol{\beta}}, \Omega_{F}\right)$ is invertible. From the direct calculation, we have $-E\left[I(Y<\infty) \Delta+\Psi_{2}^{0}(\Delta, Y, \mathbf{X}) \eta\left(\boldsymbol{\beta}_{0}^{T} \mathbf{X}\right)\left\{F_{0}(Y)-I(Y \geq y)\right\}\right]=E\left[G^{\prime}\left(\eta\left(\boldsymbol{\beta}_{0}^{T} \mathbf{X}\right) F_{0}(y)\right) \eta\left(\boldsymbol{\beta}_{0}^{T} \mathbf{X}\right) S_{c}(y \mid \mathbf{X})\right]$, 
which is negative. Thus, $\left(\Omega_{\boldsymbol{\beta}}, \Omega_{F}\right)$ can be written as the summation of an invertible operator and a compact operator. By Rudin (1973), to prove the invertibility of $\left(\Omega_{\boldsymbol{\beta}}, \Omega_{F}\right)$, it is sufficient to show that $\left(\Omega_{\boldsymbol{\beta}}, \Omega_{F}\right)$ is one-to-one. That is, if there exists some $\left(\mathbf{h}_{1}, \tilde{h}_{2}\right) \in \mathcal{S}$ such that $\Omega_{\boldsymbol{\beta}}\left(\mathbf{h}_{1}, \tilde{h}_{2}\right)=0$ and $\Omega_{F}\left(\mathbf{h}_{1}, \tilde{h}_{2}\right)=0$, we need to show $\mathbf{h}_{1}=0$ and $\tilde{h}_{2}=0$. However, we notice that according to the derivation of $\Omega$ 's, it holds that

$$
\mathbf{h}_{1}^{T} \Omega_{\boldsymbol{\beta}}\left(\mathbf{h}_{1}, \tilde{h}_{2}\right)+\int_{0}^{\infty} \Omega_{\boldsymbol{\beta}}\left(\mathbf{h}_{1}, \tilde{h}_{2}\right) \tilde{h}_{2} d F_{0}=-E\left\{l_{\boldsymbol{\beta}}\left(\boldsymbol{\beta}_{0}, F_{0}\right)^{T} \mathbf{h}_{1}+l_{F}\left(\boldsymbol{\beta}_{0}, F_{0}\right)\left[\tilde{h}_{2}\right]\right\}^{2} .
$$

We thus obtain that with probability one,

$$
l_{\boldsymbol{\beta}}\left(\boldsymbol{\beta}_{0}, F_{0}\right)^{T} \mathbf{h}_{1}+l_{F}\left(\boldsymbol{\beta}_{0}, F_{0}\right)\left[\tilde{h}_{2}\right]=0
$$

Particularly, we choose $Y=\infty$ and obtain $\mathbf{h}_{1}=0$; then we let $Y<\infty$ and $\Delta=1$ and obtain a homogeneous integral equation for $\tilde{h}_{2}$. Such an equation has one trivial solution $\tilde{h}_{2}=0$.

Finally, using the inverse of $\left(\Omega_{\boldsymbol{\beta}}, \Omega_{F}\right)$, denoted by $\left(\tilde{\Omega}_{\boldsymbol{\beta}}, \tilde{\Omega}_{F}\right)$, the equation (A.2.1) can be written as

$$
\begin{aligned}
& \sqrt{n}\left\{\left(\hat{\boldsymbol{\beta}}_{n}-\boldsymbol{\beta}_{0}\right)^{T} \mathbf{h}_{1}+\int_{0}^{\infty} \tilde{h}_{2} d\left(\hat{F}_{n}-F_{0}\right)\right\} \\
= & -\sqrt{n}\left(\mathbf{P}_{n}-\mathbf{P}\right)\left\{l_{\boldsymbol{\beta}}\left(\boldsymbol{\beta}_{0}, F_{0}\right)^{T} \tilde{\Omega}_{\boldsymbol{\beta}}\left(\mathbf{h}_{1}, \tilde{h}_{2}\right)+l_{F}\left(\boldsymbol{\beta}_{0}, F_{0}\right)^{T} \tilde{\Omega}_{F}\left(\mathbf{h}_{1}, \tilde{h}_{2}\right)\right\} \\
& +o_{p}(1)\left\{\sqrt{n}\left(\left\|\hat{\boldsymbol{\beta}}_{n}-\boldsymbol{\beta}_{0}\right\|+\left\|\hat{F}_{n}-F_{0}\right\|_{l \infty}\right)\right\},
\end{aligned}
$$

where $o_{p}(1)$ converges to zero in probability uniformly in $\left(\mathbf{h}_{1}, \tilde{h}_{2}\right) \in \mathcal{S}_{0}$, where $\mathcal{S}_{0}$ contains all $\left(\mathbf{h}_{1}, \tilde{h}_{2}\right) \in$ $\mathcal{S}$ such that $\left\|\mathbf{h}_{1}\right\| \leq 1$ and $\left\|\tilde{h}_{2}\right\|_{V} \leq 1$. This immediately implies that

$$
\sqrt{n}\left(\left\|\hat{\boldsymbol{\beta}}_{n}-\boldsymbol{\beta}_{0}\right\|+\left\|\hat{F}_{n}-F_{0}\right\|_{l \infty}\right)=O_{p}(1) .
$$

Hence,

$$
\begin{aligned}
& \sqrt{n}\left\{\left(\hat{\boldsymbol{\beta}}_{n}-\boldsymbol{\beta}_{0}\right)^{T} \mathbf{h}_{1}+\int_{0}^{\infty} \tilde{h}_{2} d\left(\hat{F}_{n}-F_{0}\right)\right\} \\
& =-\sqrt{n}\left(\mathbf{P}_{n}-\mathbf{P}\right)\left\{l_{\boldsymbol{\beta}}\left(\boldsymbol{\beta}_{0}, F_{0}\right)^{T} \tilde{\Omega}_{\boldsymbol{\beta}}\left(\mathbf{h}_{1}, \tilde{h}_{2}\right)+l_{F}\left(\boldsymbol{\beta}_{0}, F_{0}\right)^{T} \tilde{\Omega}_{F}\left(\mathbf{h}_{1}, \tilde{h}_{2}\right)\right\}+o_{p}(1) .
\end{aligned}
$$

Then $\sqrt{n}\left\{\left(\hat{\boldsymbol{\beta}}_{n}-\boldsymbol{\beta}_{0}\right)^{T} \mathbf{h}_{1}+\int_{0}^{\infty} \tilde{h}_{2} d\left(\hat{F}_{n}-F_{0}\right)\right\}$ converges weakly to a Gaussian process, denoted by $G P\left(\mathbf{h}_{1}, \tilde{h}_{2}\right)$. The covariance between $G P\left(\mathbf{h}_{1}, \tilde{h}_{2}\right)$ and $G P\left(\mathbf{h}_{1}^{*}, \tilde{h}_{2}^{*}\right)$ is given by

$$
\begin{aligned}
E[ & \left\{l_{\boldsymbol{\beta}}\left(\boldsymbol{\beta}_{0}, F_{0}\right)^{T} \tilde{\Omega}_{\boldsymbol{\beta}}\left(\mathbf{h}_{1}, \tilde{h}_{2}\right)+l_{F}\left(\boldsymbol{\beta}_{0}, F_{0}\right)\left[\tilde{\Omega}_{F}\left(\mathbf{h}_{1}, \tilde{h}_{2}\right)\right]\right\} \\
& \left.\times\left\{l_{\boldsymbol{\beta}}\left(\boldsymbol{\beta}_{0}, F_{0}\right)^{T} \tilde{\Omega}_{\boldsymbol{\beta}}\left(\mathbf{h}_{1}^{*}, \tilde{h}_{2}^{*}\right)+l_{F}\left(\boldsymbol{\beta}_{0}, F_{0}\right)\left[\tilde{\Omega}_{F}\left(\mathbf{h}_{1}^{*}, \tilde{h}_{2}^{*}\right)\right]\right\}\right] .
\end{aligned}
$$

Since for any $h_{2}, \int h_{2} d\left(\hat{F}_{n}-F_{0}\right)=\int Q_{F_{0}}\left(h_{2}\right) d\left(\hat{F}_{n}-F_{0}\right)$, the above convergence result also implies the weak convergence result in Theorem 2 . 
Specifically, if we choose in equation (A.2.2) that $\tilde{h}_{2}=0$, then we conclude that $\hat{\boldsymbol{\beta}}_{n}^{T} \mathbf{h}_{1}$ is an asymptotic linear estimator for $\boldsymbol{\beta}_{0}^{T} \mathbf{h}_{1}$ with its influence function given by

$$
l_{\boldsymbol{\beta}}\left(\boldsymbol{\beta}_{0}, F_{0}\right)^{T} \tilde{\Omega}_{\boldsymbol{\beta}}\left(\mathbf{h}_{1}, 0\right)+l_{F}\left(\boldsymbol{\beta}_{0}, F_{0}\right)\left[\tilde{\Omega}_{F}\left(\mathbf{h}_{1}, 0\right)\right]
$$

This implies that $\hat{\boldsymbol{\beta}}_{n}$ is semiparametrically efficient since the influence function is on the linear space spanned by the score functions for $\boldsymbol{\beta}_{0}$ and $F_{0}$.

Remark A.2.1. When the transformation depends on some parameter $\gamma$, the above proof can be easily adapted to this case by introducing one more parameter $\gamma$. The results hold if $\gamma_{0}$ is assumed to belong to the interior of $\Gamma,(\mathrm{C} 1)$ and $(\mathrm{C} 3)$ are replaced by assumptions $\left(\mathrm{C} 1^{\prime}\right)$ and $\left(\mathrm{C} 3^{\prime}\right)$, and the following assumption also holds:

(C5') If with probability one,

$$
G_{\gamma}^{\prime}\left(\eta\left(\boldsymbol{\beta}_{0}^{T} \mathbf{X}\right)\right) \eta^{\prime}\left(\boldsymbol{\beta}_{0}^{T} \mathbf{X}\right) \mathbf{X}^{T} \mathbf{h}_{1}+\dot{G}_{\gamma}\left(\eta\left(\boldsymbol{\beta}_{0}^{T} \mathbf{X}\right)\right) h_{3}=0
$$

where $\mathbf{h}_{1}$ and $h_{3}$ are constant vectors and $\dot{G}_{\gamma}$ denotes the derivative with respect to $\gamma$, then $\mathbf{h}_{1}=\mathbf{0}$ and $h_{3}=0$.

Note that $(\mathrm{C} 5$ ') is particularly used for proving the invertibility of $\Omega$ 's.

Remark A.2.2. The profile likelihood function can be used to give a consistent estimate for the asymptotic variance of $\hat{\boldsymbol{\beta}}_{n}$. Its justification follows from verifying all the conditions of Theorem 1 in Murphy and van der Vaart (2000). Especially, from the invertibility of $\Omega$ 's, we conclude that the information operator for $\left(\boldsymbol{\beta}_{0}, F_{0}\right)$ is invertible. Therefore, there exits a vector of functions $\mathbf{h}$ with bounded variation such that $l_{F}^{*} l_{F}\left[\int Q_{F_{0}}(\mathbf{h}) d F_{0}\right]=l_{F}^{*} l_{\boldsymbol{\beta}}$, where $l_{F}^{*}$ is the dual operator of $l_{F}$. The function $\int Q_{F_{0}}(\mathbf{h}) d F_{0}$ is called the least favorable direction in Murphy and van der Vaart (2000). We then consider the submodel $\left(\boldsymbol{\epsilon}, F_{\boldsymbol{\epsilon}}\right)$ where $\left(\boldsymbol{\epsilon}, F_{\boldsymbol{\epsilon}}\right)$, where $F_{\boldsymbol{\epsilon}}=F+(\boldsymbol{\epsilon}-\boldsymbol{\beta}) \int Q_{F}(\mathbf{h}) d F$ and $\boldsymbol{\epsilon} \in R^{d}$. It is clear that such submodel satisfies conditions (8) and (9) in Murphy and van der Vaart (2000). Furthermore, for any $\tilde{\boldsymbol{\beta}}_{n}$, we let $\tilde{F}_{n}$ be the distribution function maximizing (6) in which $\boldsymbol{\beta}=\tilde{\boldsymbol{\beta}}_{n}$. From the proof of Theorem 1 , the same arguments imply that $\tilde{F}_{n}$ uniformly converges to $F_{0}$ with probability one. We thus verify condition (10) in Murphy and van der Vaart (2000). As in the proof of Theorem 2, we linearize the likelihood function for $\tilde{F}_{n}$, which is equal to

$$
0=\mathbf{P}_{n}\left\{l_{F}\left(\tilde{\boldsymbol{\beta}}_{n}, \tilde{F}_{n}\right)\left[\int Q_{\tilde{F}_{n}}\left(h_{2}\right) d \tilde{F}_{n}\right]\right\}
$$

Following the same expansion and using the P-Donsker property as in proving Theorem 2, we obtain

$$
\sqrt{n} \int \Omega_{F}\left(0, Q_{F_{0}}\left(h_{2}\right)\right) d\left(\tilde{F}_{n}-F_{0}\right)=\sqrt{n}\left(\mathbf{P}_{n}-\mathbf{P}\right)\left\{l_{F}\left(\boldsymbol{\beta}_{0}, F_{0}\right)\left[\int Q_{F_{0}}\left[h_{2}\right] d F_{0}\right]\right\}
$$




$$
-\sqrt{n} \mathbf{P}\left[l_{F}\left(\tilde{\boldsymbol{\beta}}_{n}, F_{0}\right)\left[\int Q_{F_{0}}\left[h_{2}\right] d F_{0}\right]-l_{F}\left(\boldsymbol{\beta}_{0}, F_{0}\right)\left[\int Q_{F_{0}}\left[h_{2}\right] d F_{0}\right]\right]+o_{p}(1)
$$

From the invertibility of $\Omega_{F}(0, \cdot)$ and noting that

$$
\left|\mathbf{P}\left[l_{F}\left(\tilde{\boldsymbol{\beta}}_{n}, F_{0}\right)\left[\int Q_{F_{0}}\left[h_{2}\right] d F_{0}\right]-l_{F}\left(\boldsymbol{\beta}_{0}, F_{0}\right)\left[\int Q_{F_{0}}\left[h_{2}\right] d F_{0}\right]\right]\right| \leq O_{p}\left(\left\|\tilde{\boldsymbol{\beta}}_{n}-\boldsymbol{\beta}_{0}\right\|\right) .
$$

We obtain $\sqrt{n}\left\|\tilde{F}_{n}-F_{0}\right\|_{l^{\infty}}=O_{p}\left(\sqrt{n}+\sqrt{n}\left\|\tilde{\boldsymbol{\beta}}_{n}-\boldsymbol{\beta}_{0}\right\|\right)$. This immediately implies condition (11), i.e., the no-bias condition, in Murphy and van der Vaart (2000). Furthermore, by the same arguments as in proving Theorem 1, it is straightforward to check that the class

$$
\left\{\frac{\partial}{\partial \boldsymbol{\epsilon}} l\left(\boldsymbol{\epsilon}, F_{\boldsymbol{\epsilon}}\right):\left\|\boldsymbol{\epsilon}-\boldsymbol{\beta}_{0}\right\|+\left\|\boldsymbol{\beta}-\boldsymbol{\beta}_{0}\right\|+\left\|F-F_{0}\right\|<\delta_{0}\right\}
$$

is P-Donsker and the class

$$
\left\{\frac{\partial^{2}}{\partial \boldsymbol{\epsilon}^{2}} l\left(\boldsymbol{\epsilon}, F_{\boldsymbol{\epsilon}}\right):\left\|\boldsymbol{\epsilon}-\boldsymbol{\beta}_{0}\right\|+\left\|\boldsymbol{\beta}-\boldsymbol{\beta}_{0}\right\|+\left\|F-F_{0}\right\|<\delta_{0}\right\}
$$

is P-Glivenko-Cantelli. Thus, all the conditions in Theorem 1 of Murphy and van der Vaart (2000) hold so the results of Theorem 1 in Murphy and van der Vaart (2000) are true. One conclusion of this theorem shows the consistency of the variance estimator based on the profile likelihood function. 


\section{References}

Bennett, S. (1983). Analysis of survival data by the proportional odds model. Statistics in Medicine 2, 273-277.

Berkson, J. and Gage, R. P. (1952). Survival curve for cancer patients following treatment. Journal of the American Statistical Association 47, 501-515.

Bickel, P. J., Klaassen, C. A. J., Ritov, Y. and Wellner, J. A. (1993). Efficient and Adaptive Estimation for Semiparametric Models. Baltimore: Johns Hopkins University Press.

Chen, M. H., Harrington, D. P. and Ibrahim, J. G. (2002). Bayesian cure rate models for malignant melanoma: a case study of Eastern Cooperative Oncology Group Trial E1690. Applied Statistics 51, 135-150.

Chen, M. H., Ibrahim, J. G. and Sinha, D. (1999). A new Bayesian model for survival data with a surviving fraction. Journal of the American Statistical Association 94, 909-919.

Cheng, S. C., Wei, L. J. and Ying, Z. (1995). Analysis of transformation models with censored data. Biometrika 82, 835-845.

Cox, D. R. (1972). Regression models and life-tables (with discussion). Journal of the Royal Statistical Society, Series B 34, 187-220.

Gray, R.J. and Tsiatis, A.A. (1989). A linear rank test for use when the main interest is in differences in cure rates. Biometrics 45, 899-904.

Ibrahim, J. G., Chen, M. and Sinha, D. (2001). Bayesian Survival Analysis. New York: Springer.

Ibrahim, J.G. and Laud, P.W. (1994). A predictive approach to the analysis of designed experiments. Journal of the American Statistical Association 89, 309-319.

Kirkwood, J. M., Ibrahim, J. G., Sondak, V. K., Richards, J., Flaherty, L. E., Ernstoff, M. S., Smith, T. J., Rao, U., Steele, M. and Blum, R. H. (2000). High- and low-Dose interferon alfa-2b in high-risk melanoma: first analysis of intergroup trial E1690/S9111/C9190. Journal of Clinical Oncology 18, 2444-2458.

Kosorok, M. R., Lee, B. L., and Fine, J. P. (2004). Robust inference for proportional hazards univariate frailty regression models. Annals of Statistics 32, 1448-1491. 
Kuk, A. Y. C. and Chen, C. H. (1992). A mixture model combining logistic regression with proportional hazards regression. Biometrika 79, 531-541.

Laska, E.M. and Meisner, M.J. (1992). Nonparametric estimation and testing in a cure rate model. Biometrics 48, 1223-1234.

Lu, W. and Ying, Z. (2004). On semiparametric transformation cure models. Biometrika 91, 331-343.

Maller, R. and Zhou, X. (1996). Survival Analysis with Long-Term Survivors. New York: Wiley.

Murphy, S. A. (1994). Consistency in a proportional hazards model incorporating a random effect. Annals of Statistics 22, 712-731.

Murphy, S. A. (1995). Asymptotic theory for the frailty model. Annals of Statistics 23, 182-198.

Murphy, S. A., Rossini, A. J., and van der Vaart, A. W. (1997). Maximal likelihood estimate in the proportional odds model. Journal of the American Statistical Association 92, 968-976.

Murphy, S. A. and van der Vaart, A. W. (2000). On the profile likelihood. Journal of the American Statistical Association 95, 449-465.

Parner, E. (1998). Asymptotic theory for the correlated gamma-frailty model. Annals of Statistics 26, 183-214.

Pettitt, A. N. (1982). Inference for the linear model using a likelihood based on ranks. Journal of the Royal Statistical Society, Series B 44, 234-243.

Rudin, W. (1973). Functional Analysis. McGraw-Hill, New York.

Schwarz, G. (1978). Estimating the dimension of a model. The Annals of Statistics 6, 461-464.

Sposto, R., Sather, H.N., and Baker, S.A. (1992). A comparison of tests of the difference in the proportion of patients who are cured. Biometrics 48, 87-99.

Slud, E. and Vonta, F. (2004). Consistency of the NPML estimator in the right-censored transformation model. Scandavian Journal of Statistics 31, 21-41.

Sy, J. P. and Taylor, J. M. G. (2000). Estimation in a Cox proportional hazards cure model. Biometrics 56, 227-236. 
Taylor, J. M. G. (1995). Semi-parametric estimation in failure time mixture models. Biometrics 51, 899-907.

Tsodikov, A. (1998). A proportional hazards model taking account of long-term survivors. Biometrics 54, 1508-1516.

Yakovlev, A. Y. and Tsodikov, A. D. (1996). Stochastic Models of Tumor Latency and Their Biostatistical Applications. New Jersey: World Scientific. 


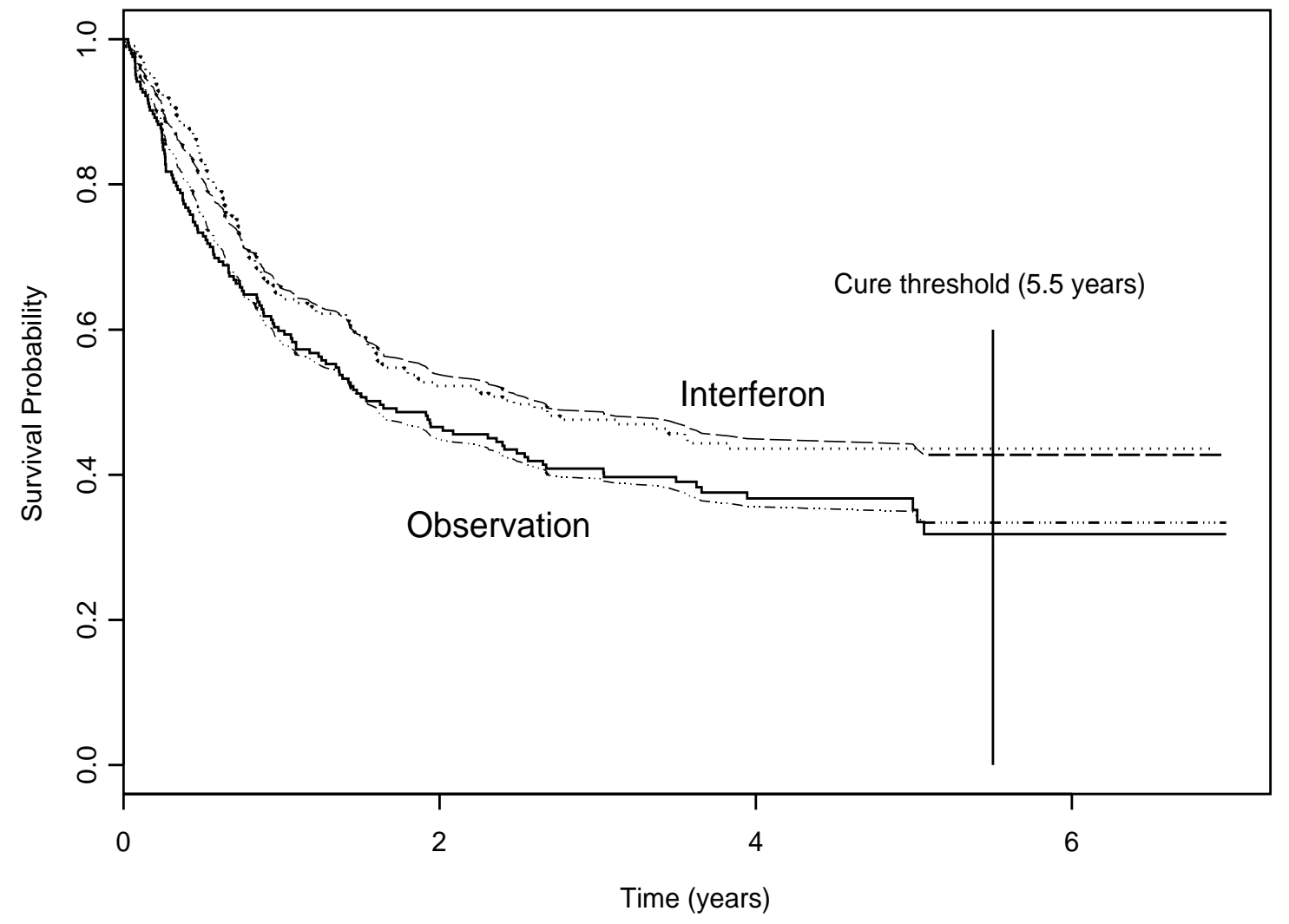

Figure 1: Kaplan-Meier curves and predicted survival curves of the interferon and observation groups in the E1690 data: the solid line and the dotted line are the Kaplan-Meier curves; the dashed line and the dot-dashed line are the predicted survival curves, respectively. 
Table 1: Simulation results from 1000 replications under the transformation $G(x)=(1+\gamma x)^{-1 / \gamma}$

\begin{tabular}{|c|c|c|c|c|c|c|c|}
\hline Model & $n$ & Parameter & True Value & Estimate & $\mathrm{SE}$ & ESE & CP (\%) \\
\hline \multirow[t]{6}{*}{$\gamma=0$} & 100 & $\overline{\beta_{0}}$ & 0.5 & 0.490 & 0.289 & 0.312 & 97.7 \\
\hline & & $\beta_{1}$ & 1 & 1.033 & 0.433 & 0.427 & 94.9 \\
\hline & & $\beta_{2}$ & -0.5 & -0.519 & 0.242 & 0.242 & 95.7 \\
\hline & 200 & $\beta_{0}$ & 0.5 & 0.502 & 0.200 & 0.218 & 96.1 \\
\hline & & $\beta_{1}$ & 1 & 1.019 & 0.300 & 0.296 & 94.6 \\
\hline & & $\beta_{2}$ & -0.5 & -0.509 & 0.167 & 0.168 & 95.9 \\
\hline \multirow[t]{6}{*}{$\gamma=0.25$} & 100 & $\beta_{0}$ & 0.5 & 0.476 & 0.341 & 0.350 & 95.6 \\
\hline & & $\beta_{1}$ & 1 & 1.036 & 0.512 & 0.493 & 94.0 \\
\hline & & $\beta_{2}$ & -0.5 & -0.490 & 0.280 & 0.281 & 96.0 \\
\hline & 200 & $\beta_{0}$ & 0.5 & 0.499 & 0.236 & 0.245 & 95.6 \\
\hline & & $\beta_{1}$ & 1 & 1.006 & 0.356 & 0.344 & 95.1 \\
\hline & & $\beta_{2}$ & -0.5 & -0.507 & 0.194 & 0.197 & 95.5 \\
\hline \multirow[t]{6}{*}{$\gamma=0.5$} & 100 & $\beta_{0}$ & 0.5 & 0.477 & 0.380 & 0.388 & 96.3 \\
\hline & & $\beta_{1}$ & 1 & 1.022 & 0.550 & 0.554 & 95.4 \\
\hline & & $\beta_{2}$ & -0.5 & -0.518 & 0.320 & 0.318 & 95.1 \\
\hline & 200 & $\beta_{0}$ & 0.5 & 0.488 & 0.271 & 0.273 & 95.5 \\
\hline & & $\beta_{1}$ & 1 & 1.015 & 0.400 & 0.388 & 94.9 \\
\hline & & $\beta_{2}$ & -0.5 & -0.505 & 0.225 & 0.222 & 95.1 \\
\hline \multirow[t]{6}{*}{$\gamma=0.75$} & 100 & $\beta_{0}$ & 0.5 & 0.487 & 0.410 & 0.423 & 95.7 \\
\hline & & $\beta_{1}$ & 1 & 0.995 & 0.601 & 0.607 & 95.1 \\
\hline & & $\beta_{2}$ & -0.5 & -0.491 & 0.359 & 0.348 & 94.2 \\
\hline & 200 & $\beta_{0}$ & 0.5 & 0.486 & 0.284 & 0.298 & 96.5 \\
\hline & & $\beta_{1}$ & 1 & 1.022 & 0.426 & 0.425 & 94.7 \\
\hline & & $\beta_{2}$ & -0.5 & -0.494 & 0.241 & 0.244 & 95.4 \\
\hline \multirow[t]{6}{*}{$\gamma=1$} & 100 & $\beta_{0}$ & 0.5 & 0.455 & 0.426 & 0.458 & 96.7 \\
\hline & & $\beta_{1}$ & 1 & 1.043 & 0.637 & 0.658 & 96.1 \\
\hline & & $\beta_{2}$ & -0.5 & -0.498 & 0.375 & 0.378 & 95.4 \\
\hline & 200 & $\beta_{0}$ & 0.5 & 0.482 & 0.310 & 0.321 & 95.4 \\
\hline & & $\beta_{1}$ & 1 & 1.015 & 0.458 & 0.460 & 94.8 \\
\hline & & $\beta_{2}$ & -0.5 & -0.502 & 0.258 & 0.264 & 95.8 \\
\hline
\end{tabular}


Table 2: Simulation results from 1000 replications under the transformation $G(x)=\exp \left[-\left\{(1+x)^{\gamma}-\right.\right.$ $1\} / \gamma$ ]

\begin{tabular}{|c|c|c|c|c|c|c|c|}
\hline Model & $n$ & Parameter & True Value & Estimate & $\mathrm{SE}$ & ESE & $\mathrm{CP}(\%)$ \\
\hline \multirow{6}{*}{$\gamma=0$} & \multirow[t]{3}{*}{100} & $\beta_{0}$ & 0.5 & 0.465 & 0.442 & 0.458 & 96.6 \\
\hline & & $\beta_{1}$ & 1 & 1.026 & 0.632 & 0.658 & 96.4 \\
\hline & & $\beta_{2}$ & -0.5 & -0.510 & 0.387 & 0.378 & 94.8 \\
\hline & \multirow[t]{3}{*}{200} & $\beta_{0}$ & 0.5 & 0.498 & 0.318 & 0.321 & 95.4 \\
\hline & & $\beta_{1}$ & 1 & 0.995 & 0.474 & 0.461 & 93.9 \\
\hline & & $\beta_{2}$ & -0.5 & -0.504 & 0.263 & 0.264 & 95.0 \\
\hline \multirow[t]{6}{*}{$\gamma=0.25$} & \multirow[t]{3}{*}{100} & $\beta_{0}$ & 0.5 & 0.500 & 0.391 & 0.406 & 95.2 \\
\hline & & $\beta_{1}$ & 1 & 0.994 & 0.568 & 0.585 & 96.3 \\
\hline & & $\beta_{2}$ & -0.5 & -0.501 & 0.328 & 0.335 & 95.7 \\
\hline & \multirow[t]{3}{*}{200} & $\beta_{0}$ & 0.5 & 0.489 & 0.283 & 0.285 & 94.8 \\
\hline & & $\beta_{1}$ & 1 & 1.010 & 0.397 & 0.409 & 95.9 \\
\hline & & $\beta_{2}$ & -0.5 & -0.502 & 0.237 & 0.235 & 94.7 \\
\hline \multirow[t]{6}{*}{$\gamma=0.5$} & \multirow[t]{3}{*}{100} & $\beta_{0}$ & 0.5 & 0.459 & 0.356 & 0.364 & 95.8 \\
\hline & & $\beta_{1}$ & 1 & 1.081 & 0.545 & 0.523 & 94.7 \\
\hline & & $\beta_{2}$ & -0.5 & -0.500 & 0.297 & 0.299 & 95.8 \\
\hline & \multirow[t]{3}{*}{200} & $\beta_{0}$ & 0.5 & 0.502 & 0.247 & 0.256 & 96.3 \\
\hline & & $\beta_{1}$ & 1 & 1.005 & 0.360 & 0.365 & 95.4 \\
\hline & & $\beta_{2}$ & -0.5 & -0.502 & 0.214 & 0.209 & 93.6 \\
\hline \multirow[t]{6}{*}{$\gamma=0.75$} & \multirow[t]{3}{*}{100} & $\beta_{0}$ & 0.5 & 0.471 & 0.318 & 0.332 & 96.8 \\
\hline & & $\beta_{1}$ & 1 & 1.069 & 0.479 & 0.469 & 93.9 \\
\hline & & $\beta_{2}$ & -0.5 & -0.505 & 0.264 & 0.267 & 95.3 \\
\hline & \multirow[t]{3}{*}{200} & $\beta_{0}$ & 0.5 & 0.506 & 0.228 & 0.233 & 95.8 \\
\hline & & $\beta_{1}$ & 1 & 1.000 & 0.327 & 0.326 & 94.8 \\
\hline & & $\beta_{2}$ & -0.5 & -0.500 & 0.192 & 0.187 & 94.2 \\
\hline \multirow[t]{6}{*}{$\gamma=1$} & \multirow[t]{3}{*}{100} & $\beta_{0}$ & 0.5 & 0.509 & 0.289 & 0.314 & 97.8 \\
\hline & & $\beta_{1}$ & 1 & 1.008 & 0.419 & 0.423 & 95.5 \\
\hline & & $\beta_{2}$ & -0.5 & -0.516 & 0.245 & 0.242 & 94.2 \\
\hline & \multirow[t]{3}{*}{200} & $\beta_{0}$ & 0.5 & 0.508 & 0.205 & 0.219 & 97.1 \\
\hline & & $\beta_{1}$ & 1 & 1.010 & 0.296 & 0.296 & 95.2 \\
\hline & & $\beta_{2}$ & -0.5 & -0.508 & 0.172 & 0.168 & 94.1 \\
\hline
\end{tabular}


Table 3: Simulation results from 1000 replications under misspecified transformation $(n=100)$

\begin{tabular}{|c|c|c|c|c|c|c|}
\hline Model & Parameter & True Value & Estimate & $\mathrm{SE}$ & $\mathrm{ESE}$ & $\mathrm{CP}(\%)$ \\
\hline \multicolumn{7}{|c|}{ True Transformation: $G(x)=(1+x / 2)^{-2}$} \\
\hline \multirow[t]{3}{*}{ Proportional Hazards Model } & $\beta_{0}$ & 0.5 & 0.165 & 0.290 & 0.311 & 82.6 \\
\hline & $\beta_{1}$ & 1 & 0.799 & 0.450 & 0.446 & 92.5 \\
\hline & $\beta_{2}$ & -0.5 & -0.404 & 0.248 & 0.255 & 94.2 \\
\hline \multirow[t]{3}{*}{ Proportional Odds Model } & $\beta_{0}$ & 0.5 & 0.818 & 0.456 & 0.466 & 90.8 \\
\hline & $\beta_{1}$ & 1 & 1.240 & 0.672 & 0.654 & 93.0 \\
\hline & $\beta_{2}$ & -0.5 & -0.578 & 0.375 & 0.373 & 95.1 \\
\hline \multicolumn{7}{|c|}{ True Transformation: $G(x)=\exp \left[-2\left\{(1+x)^{1 / 2}-1\right\}\right]$} \\
\hline \multirow[t]{3}{*}{ Proportional Hazards Model } & $\beta_{0}$ & 0.5 & 0.189 & 0.304 & 0.311 & 84.1 \\
\hline & $\beta_{1}$ & 1 & 0.868 & 0.464 & 0.442 & 91.9 \\
\hline & $\beta_{2}$ & -0.5 & -0.411 & 0.254 & 0.252 & 92.7 \\
\hline \multirow[t]{3}{*}{ Proportional Odds Model } & $\beta_{0}$ & 0.5 & 0.960 & 0.463 & 0.472 & 84.4 \\
\hline & $\beta_{1}$ & 1 & 1.205 & 0.650 & 0.652 & 94.8 \\
\hline & $\beta_{2}$ & -0.5 & -0.606 & 0.363 & 0.373 & 95.0 \\
\hline
\end{tabular}


Table 4: Estimates of regression coefficients in the proportional hazards cure model for the E1690 data

\begin{tabular}{|c|c|c|c|c|}
\hline Cure Threshold & Covariate & Estimate & Std. Err. & $p$-value \\
\hline \multirow{5}{*}{5.1 years } & Intercept & -0.7977 & 0.3147 & 0.0113 \\
\hline & Treatment & -0.2200 & 0.1298 & 0.0901 \\
\hline & Age & 0.0115 & 0.0050 & 0.0220 \\
\hline & Sex & -0.2209 & 0.1371 & 0.1072 \\
\hline & Nodal category & 0.5519 & 0.1599 & 0.0006 \\
\hline \multirow[t]{5}{*}{5.5 years } & Intercept & -0.8027 & 0.3156 & 0.0110 \\
\hline & Treatment & -0.2197 & 0.1300 & 0.0911 \\
\hline & Age & 0.0115 & 0.0050 & 0.0225 \\
\hline & Sex & -0.2208 & 0.1374 & 0.1081 \\
\hline & Nodal category & 0.5520 & 0.1603 & 0.0006 \\
\hline \multirow[t]{5}{*}{6 years } & Intercept & -0.7988 & 0.3151 & 0.0112 \\
\hline & Treatment & -0.2199 & 0.1298 & 0.0902 \\
\hline & Age & 0.0115 & 0.0050 & 0.0220 \\
\hline & Sex & -0.2209 & 0.1372 & 0.1074 \\
\hline & Nodal category & 0.5519 & 0.1600 & 0.0006 \\
\hline \multirow[t]{5}{*}{6.5 years } & Intercept & -0.7969 & 0.3147 & 0.0113 \\
\hline & Treatment & -0.2200 & 0.1297 & 0.0898 \\
\hline & Age & 0.0115 & 0.0050 & 0.0219 \\
\hline & Sex & -0.2210 & 0.1371 & 0.1070 \\
\hline & Nodal category & 0.5518 & 0.1599 & 0.0006 \\
\hline \multirow[t]{5}{*}{7 years } & Intercept & -0.7972 & 0.3148 & 0.0113 \\
\hline & Treatment & -0.2200 & 0.1297 & 0.0898 \\
\hline & Age & 0.0115 & 0.0050 & 0.0219 \\
\hline & Sex & -0.2209 & 0.1371 & 0.1071 \\
\hline & Nodal category & 0.5518 & 0.1599 & 0.0006 \\
\hline
\end{tabular}



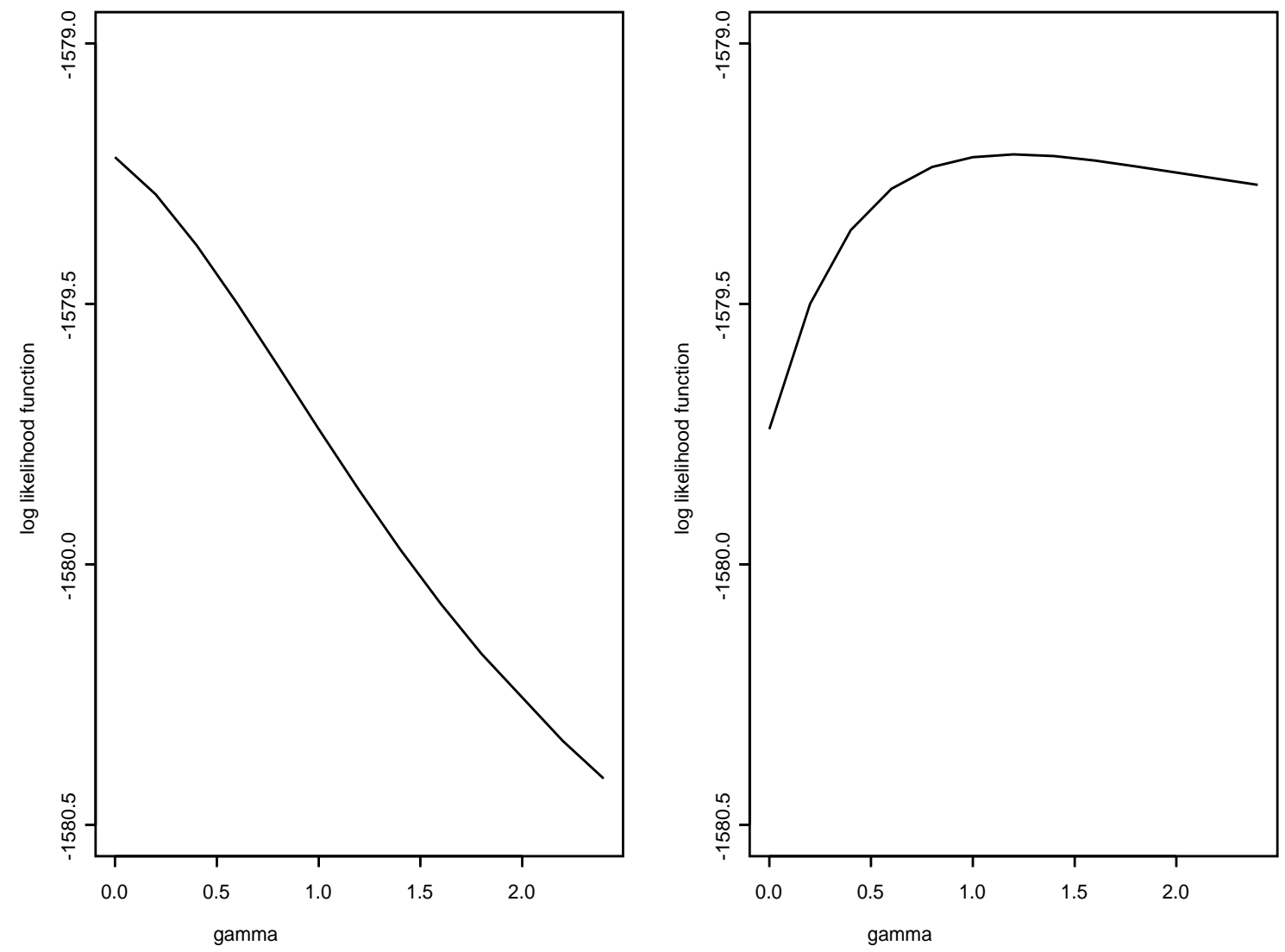

Figure 2: The observed log-likelihood functions from different transformations in the E1690 data: the left plot is the log-likelihood functions from transformations $G(x)=(1+\gamma x)^{-1 / \gamma}$; the right plot is the $\log$-likelihood functions from transformations $G(x)=\exp \left\{-\left((1+x)^{\gamma}-1\right) / \gamma\right\}$. 